\title{
(Sub)millimetre interferometric imaging of a sample of COSMOS/AzTEC submillimetre galaxies
}

\section{The spatial extent of the radio-emitting regions ${ }^{\star}$}

\author{
O. Miettinen ${ }^{1}$, M. Novak ${ }^{1}$, V. Smolčić ${ }^{1}$, E. Schinnerer ${ }^{2}$, M. Sargent ${ }^{3}$, E. J. Murphy ${ }^{4}$, M. Aravena ${ }^{5}$, M. Bondi ${ }^{6}$, \\ C. L. Carilli ${ }^{7,8}$, A. Karim ${ }^{9}$, M. Salvato ${ }^{10}$, and G. Zamorani ${ }^{11}$ \\ ${ }^{1}$ Department of Physics, University of Zagreb, Bijenička cesta 32, 10000 Zagreb, Croatia \\ e-mail: oskari@phy.hr \\ 2 Max-Planck-Institut für Astronomie, Königstuhl 17, 69117 Heidelberg, Germany \\ 3 Astronomy Centre, Department of Physics and Astronomy, University of Sussex, Brighton, BN1 9QH, UK \\ 4 Infrared Processing \& Analysis Center, MS 100-22, California Institute of Technology, Pasadena, CA 91125, USA \\ 5 Núcleo de Astronomía, Facultad de Ingeniería, Universidad Diego Portales, Av. Ejército 441, Santiago, Chile \\ ${ }^{6}$ Istituto di Radioastronomia - INAF, via Gobetti 101, 40129 Bologna, Italy \\ 7 National Radio Astronomy Observatory, PO Box 0, Socorro, NM 87801-0387, USA \\ 8 Astrophysics Group, Cavendish Laboratory, JJ Thomson Avenue, Cambridge CB3 OHE, UK \\ 9 Argelander-Institut für Astronomie, Universität Bonn, Auf dem Hügel 71, 53121 Bonn, Germany \\ 10 Max-Planck-Institut für extraterrestrische Physik, Giessenbachstrasse 1, 85748 Garching bei München, Germany \\ 11 INAF-Osservatorio Astronomico di Bologna, via Ranzani 1, 40127 Bologna, Italy
}

Received 23 May 2015 / Accepted 22 September 2015

\begin{abstract}
Radio emission at centimetre wavelengths from highly star-forming galaxies, like submillimetre galaxies (SMGs), is dominated by synchrotron radiation arising from supernova activity. Hence, radio continuum imaging has the potential to determine the spatial extent of star formation in these types of galaxies. Using deep, high-resolution $\left(1 \sigma=2.3 \mu \mathrm{Jy} \mathrm{beam}{ }^{-1} ; 0\right.$ ' 75$)$ centimetre radio-continuum observations taken by the Karl G. Jansky Very Large Array (VLA)-COSMOS $3 \mathrm{GHz}$ Large Project, we studied the radio-emitting sizes of a flux-limited sample of SMGs in the COSMOS field. The target SMGs were originally discovered in a $1.1 \mathrm{~mm}$ continuum survey carried out with the AzTEC bolometer, and followed up with higher resolution interferometric (sub)millimetre continuum observations. Of the $39 \mathrm{SMGs}$ studied here, $3 \mathrm{GHz}$ emission was detected towards 18 of them $(\sim 46 \pm 11 \%)$ with signal-to-noise ratios in the range of $S / N=4.2-37.4$. Towards four SMGs (AzTEC2, 5, 8, and 11), we detected two separate $3 \mathrm{GHz}$ sources with projected separations of $\sim 1^{\prime \prime}$. 5-6.' 6 , but they might be physically related in only one or two cases (AzTEC2 and 11). Using two-dimensional elliptical Gaussian fits, we derived a median deconvolved major axis FWHM size of 0,'54 \pm 0 .' 11 for our 18 SMGs detected at $3 \mathrm{GHz}$. For the 15 SMGs with known redshift we derived a median linear major axis FWHM of $4.2 \pm 0.9 \mathrm{kpc}$. No clear correlation was found between the radio-emitting size and the $3 \mathrm{GHz}$ or submm flux density, or the redshift of the SMG. However, there is a hint of larger radio sizes at $z \sim 2.5-5$ compared to lower redshifts. The sizes we derived are consistent with previous SMG sizes measured at $1.4 \mathrm{GHz}$ and in mid- $J \mathrm{CO}$ emission, but significantly larger than those seen in the (sub)mm continuum emission (typically probing the rest-frame far-infrared with median FWHM sizes of only $\sim 1.5-2.5 \mathrm{kpc}$ ). One possible scenario is that SMGs have i) an extended gas component with a low dust temperature, which can be traced by low- to mid- $J$ CO line emission and radio continuum emission; and ii) a warmer, compact starburst region giving rise to the high-excitation line emission of $\mathrm{CO}$, which could dominate the dust continuum size measurements. Because of the rapid cooling of cosmic-ray electrons in dense starburst galaxies $\left(\sim 10^{4}-10^{5} \mathrm{yr}\right)$, the more extended synchrotron radio-emitting size being a result of cosmic-ray diffusion seems unlikely. Instead, if SMGs are driven by galaxy mergers - a process where the galactic magnetic fields can be pulled out to larger spatial scales - the radio synchrotron emission might arise from more extended magnetised interstellar medium around the starburst region.
\end{abstract}

Key words. galaxies: evolution - galaxies: formation - galaxies: starburst - galaxies: star formation - radio continuum: galaxies submillimeter: galaxies

\section{Introduction}

Submillimetre galaxies (SMGs; e.g. Smail et al. 1997; Hughes et al. 1998; Barger et al. 1998) are a population of distant galaxies where star formation is heavily obscured by the dusty interstellar medium (ISM). The star formation rates (SFRs) in SMGs lie in the range of $\sim 10^{2}-10^{3} M_{\odot} \mathrm{yr}^{-1}$, and hence these galaxies stand out as the most intense starbursts in the

* Appendices are available in electronic form at http://www. aanda.org universe (for reviews, see Blain et al. 2002; Casey et al. 2014). As the potential precursors to the present-day massive elliptical galaxies, SMGs have become one of the primary targets for understanding galaxy evolution across cosmic time (e.g. Swinbank et al. 2006; Fu et al. 2013; Toft et al. 2014; Simpson et al. 2014). In the context of this evolutionary connection, determining the sizes and size evolution of SMGs is crucial.

Nearly all of the cm-wavelength radio emission from starforming galaxies, such as SMGs, is non-thermal synchrotron 
radiation from relativistic electrons accelerated in supernova (SN) remnants produced by the short-lived, high-mass OB-type stars $\left(M \gtrsim 8 M_{\odot}\right.$; main-sequence lifetime $\left.\tau_{\mathrm{MS}} \lesssim 30 \mathrm{Myr}\right)$. Because SNe trace the recent/on-going star formation, the radio synchrotron emission has the potential to trace the spatial scales on which star formation is occurring. This connection between radio emission and star formation is strongly supported by the close infrared (IR)-radio correlation observed in galaxies (e.g. Helou et al. 1985; Beck \& Golla 1988; Xu et al. 1992; Condon 1992; Yun et al. 2001; Bell 2003; Tabatabaei et al. 2007; Murphy et al. 2008; Sargent et al. 2010; Morić et al. 2010; Dumas et al. 2011). On the basis of this correlation, the IR-emitting region of a star-forming galaxy is expected to be comparable in size to that of radio continuum emission. However, the most recent studies of the sizes of IR-emitting regions of SMGs based on continuum imaging observations with the Atacama Large Millimetre/submillimetre Array (ALMA) show that these are significantly smaller than SMG radio sizes presented in the literature (Simpson et al. 2015a; Ikarashi et al. 2015). A possible explanation for this discrepancy, as suggested by Simpson et al. (2015a), is cosmic ray (CR) diffusion in the galactic magnetic field away from their acceleration site, which would render larger radio sizes. To test this further here we present a study of radio sizes of SMGs from a well selected sample of SMGs in the Cosmic Evolution Survey (COSMOS; Scoville et al. 2007) deep field using radio data from the Karl G. Jansky Very Large Array (VLA)-COSMOS $3 \mathrm{GHz}$ Large Project ( $1 \sigma$ noise of $2.3 \mu \mathrm{Jy} \mathrm{beam}^{-1}$, angular resolution 0.75 ; Smolčić et al., in prep.). We describe the SMG sample and the employed VLA data in detail in Sect. 2. The $3 \mathrm{GHz}$ images are presented in Sect. 3, and the analysis (size measurements and radio spectral indices) are presented in Sect. 4. We compare our results with literature studies in Sect. 5, discuss the results in Sect. 6, and summarise the main results of the paper in Sect. 7.

To be consistent with the most recent results from the Planck mission (Planck Collaboration XIII 2015), the cosmology adopted in the present work corresponds to the flat $\Lambda \mathrm{CDM}$ universe with the dark energy density $\Omega_{\Lambda}=0.692$, total (dark+luminous baryonic) matter density $\Omega_{\mathrm{m}}=0.308$, and a Hubble constant of $H_{0}=67.8 \mathrm{~km} \mathrm{~s}^{-1} \mathrm{Mpc}^{-1}$.

\section{Data}

\subsection{Source sample}

The target SMGs of the present study - AzTEC1-30 - were originally discovered in the JCMT/AzTEC $1.1 \mathrm{~mm}$ continuum survey $\left(18^{\prime \prime}\right.$ resolution) towards a COSMOS subfield $\left(0.15 \mathrm{deg}^{2}\right.$ in size) by Scott et al. (2008). The signal-to-noise ratios $(\mathrm{S} / \mathrm{N})$ of these SMGs were found to be in the range of $S / N_{1.1 \mathrm{~mm}}=4.0-8.3$ (see Table 1 in Scott et al. 2008). The 15 brightest sources, AzTEC1-15 $\left(S / N_{1.1 \mathrm{~mm}} \geq 4.6\right)$, were imaged (and detected) with the Submillimetre Array (SMA) at $890 \mu \mathrm{m}$ (2" resolution) by Younger et al. (2007, 2009). More recently, AzTEC16-30 $\left(S / N_{1.1 \mathrm{~mm}}=4.0-4.5\right)$ were imaged with the Plateau de Bure Interferometer $(\mathrm{PdBI})$ at $1.3 \mathrm{~mm}\left(\sim 1^{\prime \prime} .8\right.$ resolution $)$ by Miettinen et al. (2015). These interferometric follow-up studies have allowed us to accurately determine the position of the actual SMGs giving rise to the millimetre continuum emission seen in the single-dish AzTEC maps and, in eight cases, to resolve the single-dish emission into multiple (two to three) components (at $\sim 2^{\prime \prime}$ resolution). This way, we can reliably identify the correct $3 \mathrm{GHz}$ counterparts of the target SMGs. We note that even the faintest component in our source sample (AzTEC26b) has a

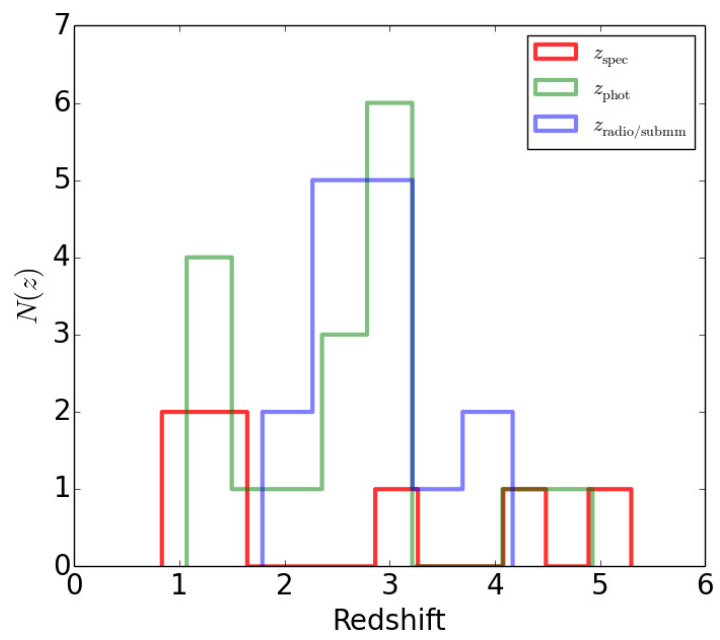

Fig. 1. Redshift distribution of the target SMGs divided into three subsamples: the red and green histograms show the spectroscopic and photometric redshifts, respectively, while the blue histogram shows the redshift values derived from the radio-to-submm spectral index (see Table 1). The redshift bins have a width of $\Delta z=0.4$. The lower redshift limits were placed in the bins corresponding to those values.

$1.3 \mathrm{~mm}$ flux density of $0.9 \mathrm{mJy}$, which corresponds to $\sim 4 \mathrm{mJy}$ at the observed-frame $850 \mu \mathrm{m}$ (assuming a dust emissivity index of $\beta=1.5$; see Miettinen et al. 2015), and hence can be considered an SMG (cf. the classic SMG threshold of $S_{850 \mu \mathrm{m}}>5 \mathrm{mJy}$ refers to bright SMGs e.g. Hainline et al. 2009; González et al. 2011). We also note that none of these SMGs has been detected in X-rays, and hence they do not appear to harbour any strong active galactic nucleus (AGN; a typical $3 \sigma$ upper limit to the flux density in the $0.5-2 \mathrm{keV}$ band data of the Chandra COSMOS Legacy Survey is $<6 \times 10^{-16} \mathrm{erg} \mathrm{cm}^{-2} \mathrm{~s}^{-1}$, F. Civano et al., in prep.). This suggests that the observed radio emission from our SMGs is predominantly powered by star formation. This is further supported by the fact that none of our SMGs were detected with the Very Long Baseline Array (VLBA) observations at a high, milliarcsec resolution at $1.4 \mathrm{GHz}$ (Herrera Ruiz et al., in prep.), yielding a $3 \sigma$ flux density upper limit to $S_{1.4 \mathrm{GHz}}^{\mathrm{VLBA}}$ of $<60 \mu \mathrm{Jy}_{\text {beam }^{-1}}$. $^{1}$

Our sample of 39 SMGs is listed in Table 1. The coordinates given in the table correspond to the (sub)mm peak positions determined in the aforementioned SMA and PdBI studies. Table 1 also provides the source redshifts that are based on spectroscopic measurements (seven sources), optical to nearinfrared (NIR) spectral energy distribution fitting (i.e. photometric redshift; 17 sources), and radio/submm flux density ratios (15 sources). The redshift distribution is shown in Fig. 1. We refer to Miettinen et al. (2015, and references therein) for further details and discussion on the redshifts of our SMGs.

\subsection{VLA $3 \mathrm{GHz}$ radio continuum data}

The observations used in the present paper were taken by the VLA-COSMOS $3 \mathrm{GHz}$ Large Project (PI: Smolčić; Smolčić et al., in prep.). Details of the observations, data reduction, and imaging can be found in Novak et al. (2015), Smolčić et al. (2015a), and Smolčić et al. (in prep.). In the present paper,

\footnotetext{
1 As described in Appendix A, we have detected two $3 \mathrm{GHz}$ sources towards AzTEC8. The western radio source is associated with our target SMG, while the eastern $3 \mathrm{GHz}$ source, physically unrelated to the SMG, is also detected at $1.4 \mathrm{GHz}$ with the VLBA.
} 
Table 1. Source list.

\begin{tabular}{|c|c|c|c|c|}
\hline Source ID & $\begin{array}{c}\alpha_{2000.0} \\
{[\mathrm{~h}: \mathrm{m}: \mathrm{s}]}\end{array}$ & $\begin{array}{l}\delta_{2000.0} \\
{\left[{ }^{\circ}:^{\prime \prime}:^{\prime \prime}\right]}\end{array}$ & $\overline{\text { Redshift }^{a}}$ & $\bar{z}$ reference $^{a}$ \\
\hline AzTEC1 & 095942.86 & +022938.2 & $z_{\text {spec }}=4.3415$ & 1 \\
\hline AzTEC2 & 100008.05 & +022612.2 & $z_{\text {spec }}=1.125$ & 2 \\
\hline AzTEC3 & 100020.70 & +023520.5 & $z_{\text {spec }}=5.298$ & 3 \\
\hline AzTEC4 & 095931.72 & +023044.0 & $z_{\text {phot }}=4.93_{-11}^{+0.43}$ & 4 \\
\hline AzTEC5 & 100019.75 & +023204.4 & $z_{\text {phot }}=3.05_{-0.28}^{+0.31}$ & 4 \\
\hline AzTEC6 & 100006.50 & +023837.7 & $z_{\text {radio/submm }}>3.52$ & 5 \\
\hline AzTEC7 & 100018.06 & +024830.5 & $z_{\text {phot }}=2.30 \pm 0.10$ & 4 \\
\hline AzTEC8 & 095959.34 & +023441.0 & $z_{\mathrm{spec}}=3.179$ & 6 \\
\hline AzTEC9 & 095957.25 & +022730.6 & $z_{\text {phot }}=1.07_{-0.10}^{+0.11}$ & 4 \\
\hline AzTEC10 & 095930.76 & +024033.9 & $z_{\text {phot }}=2.79_{-1.29}^{+1.86}$ & 4 \\
\hline AzTEC11-N ${ }^{b}$ & 100008.91 & +024009.6 & $z_{\text {spec }}=1.599$ & 7 \\
\hline AzTEC $11-S^{b}$ & 100008.94 & +024012.3 & $z_{\text {spec }}=1.599$ & 7 \\
\hline AzTEC12 & 100035.29 & +024353.4 & $z_{\text {phot }}=2.54_{-0.33}^{+0.13}$ & 4 \\
\hline AzTEC13 & 095937.05 & +023320.0 & $z_{\text {radio/submm }}>4.07$ & 5 \\
\hline AzTEC14-E ${ }^{c}$ & 100010.03 & +023014.7 & $z_{\text {radio/submm }}>2.95$ & 5 \\
\hline AzTEC14-W ${ }^{c}$ & 100009.63 & +023018.0 & $z_{\text {phot }}=1.30_{-0.36}^{+0.12}$ & 4 \\
\hline AzTEC15 & 100012.89 & +023435.7 & $z_{\text {phot }}=3.17_{-0.37}^{+0.29}$ & 4 \\
\hline AzTEC16 & 095950.069 & +024424.50 & $z_{\text {radio/submm }}>2.42$ & 5 \\
\hline AzTEC17a & 095939.194 & +023403.83 & $z_{\mathrm{spec}}=0.834$ & 7 \\
\hline AzTEC17b & 095938.904 & +023404.69 & $z_{\text {phot }}=4.14_{-1.73}^{+0.87}$ & 5 \\
\hline AzTEC18 & 095942.607 & +023536.96 & $z_{\text {phot }}=3.00_{-0.17}^{+0.19}$ & 5 \\
\hline AzTEC19a & 100028.735 & +023203.84 & $z_{\text {phot }}=3.20_{-0.45}^{+0.18}$ & 5 \\
\hline AzTEC19b & 100029.256 & +023209.82 & $z_{\text {phot }}=1.11 \pm 0.10$ & 5 \\
\hline AzTEC20 & 100020.251 & +024121.66 & $z_{\text {radio/submm }}>2.35$ & 5 \\
\hline AzTEC21a & 100002.558 & +024641.74 & $z_{\text {phot }}=2.60_{-0.17}^{+0.18}$ & 5 \\
\hline AzTEC21b & 100002.710 & +024644.51 & $z_{\text {phot }}=2.80_{-0.16}^{+0.17}$ & 5 \\
\hline AzTEC21c & 100002.856 & +024640.80 & $z_{\text {radio/submm }}>1.93$ & 5 \\
\hline AzTEC22 & 095950.681 & +022819.06 & $z_{\text {radio/submm }}>3.00$ & 5 \\
\hline AzTEC 23 & 095931.399 & +023604.61 & $z_{\text {phot }}=1.60_{-0.50}^{+0.28}$ & 5 \\
\hline AzTEC24a & 100038.969 & +023833.90 & $z_{\text {radio/submm }}>2.35$ & 5 \\
\hline AzTEC24b & 100039.410 & +023846.97 & $z_{\text {radio/submm }}>2.28$ & 5 \\
\hline AzTEC24c & 100039.194 & +023854.46 & $z_{\text {radio/submm }}>3.17$ & 5 \\
\hline $\mathrm{AzTEC} 25^{d}$ & 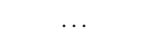 & 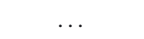 & $\cdots$ & $\cdots$ \\
\hline AzTEC26a & 095959.386 & +023815.36 & $z_{\text {phot }}=2.50_{-0.14}^{+0.24}$ & 5 \\
\hline AzTEC26b & 095959.657 & +023821.08 & $z_{\text {radio/submm }}>1.79$ & 5 \\
\hline AzTEC27 & 100039.211 & +024052.18 & $z_{\text {radio/submm }}>4.17$ & 5 \\
\hline AzTEC28 & 100004.680 & +023037.30 & $z_{\text {radio/submm }}>3.11$ & 5 \\
\hline AzTEC29a & 100026.351 & +023744.15 & $z_{\text {radio/submm }}>2.96$ & 5 \\
\hline AzTEC29b & 100026.561 & +023805.14 & $z_{\text {phot }}=1.45_{-0.38}^{+0.79}$ & 5 \\
\hline AzTEC30 & 100003.552 & +023300.94 & $z_{\text {radio/submm }}>2.51$ & 5 \\
\hline
\end{tabular}

Notes. The coordinates given in Cols. (2) and (3) for AzTEC1-15 refer to the SMA $890 \mu \mathrm{m}$ peak position (Younger et al. 2007, 2009), while those for AzTEC16-30 are the PdBI $1.3 \mathrm{~mm}$ peak positions (Miettinen et al. 2015). ${ }^{(a)}$ The $z_{\text {spec }}, z_{\text {phot }}$, and $z_{\text {radio/submm }}$ values are the spectroscopic redshift, optical-NIR photometric redshift, and the redshift derived using the Carilli-Yun redshift indicator (Carilli \& Yun 1999, 2000). The $z$ references in the last column are as follows: $1=$ Yun et al. (2015); 2 = Baloković et al. (in prep.); 3 = Riechers et al. (2010) and Capak et al. (2011); 4 = Smolčić et al. (2012); $5=$ Miettinen et al. (2015); $6=$ Riechers et al. (in prep.); $7=$ M. Salvato et al. (in prep.) (b) AzTEC11 was resolved into two $890 \mu \mathrm{m}$ sources (N and S) by Younger et al. (2009). The two components are probably physically related, i.e. are at the same redshift (see Appendix A). ${ }^{(c)}$ AzTEC14 was resolved into two $890 \mu \mathrm{m}$ sources (E and W) by Younger et al. (2009). The eastern component appears to lie at a higher redshift than the western one (Smolčić et al. 2012). ${ }^{(d)}$ AzTEC25 was not detected in the $1.3 \mathrm{~mm}$ PdBI observations (Miettinen et al. 2015).

we employ - for the first time - the final, full $3 \mathrm{GHz}$ mosaic imaging of COSMOS (192 pointings in total). Briefly, these Sband observations were carried out with the VLA of the NRAO ${ }^{2}$ in its $\mathrm{A}$ and $\mathrm{C}$ configurations (maximum baseline of $36.4 \mathrm{~km}$ and $3.4 \mathrm{~km}$, respectively) between 2012 and 2014. The $2 \mathrm{GHz}$ bandwidth ( 2 basebands of $1 \mathrm{GHz}$ each) used was divided into 16 sub-bands/spectral windows (SPWs) each with a $128 \mathrm{MHz}$

2 The National Radio Astronomy Observatory is a facility of the National Science Foundation operated under cooperative agreement by Associated Universities, Inc. bandwidth. Each SPW was subdivided into 64 spectral channels with a width of $2 \mathrm{MHz}$. The data were calibrated using the AIPSLite data reduction pipeline, which is an extension of NRAO's Astronomical Image Processing System (AIPS) ${ }^{3}$ package (Bourke et al. 2014; Mooley et al., in prep.), and adapted for the VLA-COSMOS $3 \mathrm{GHz}$ Large Project (for details, see Smolčić et al., in prep.). Further editing, flagging, and imaging was done using the Common Astronomy Software Applications package (CASA ${ }^{4}$; McMullin et al. 2007). To reduce sidelobes and artefacts in the data, phase solutions obtained from selfcalibration with the bright quasar J1024-0052 were applied on each pointing. Every field was cleaned down to $5 \sigma$, and further cleaned down to $1.5 \sigma$ using manually defined masks around the sources.

The data used here were imaged using the multi-scale multifrequency synthesis (MS-MFS) method (Rau \& Cornwell 2011). Briggs or robust weighting was applied to the calibrated visibilities with a robust value of 0.5 . Considering the aim of the present study (i.e. measuring the $3 \mathrm{GHz}$ sizes of our SMGs), the main advantage of MS-MFS is that the final image resolution is not determined by the lowest frequency of the bandwidth used because all the SPWs are used in the image deconvolution. A Gaussian $u-v$ tapering was applied on each pointing using their own Gaussian beam size (Full Width at Half Maximum or FWHM). The final mosaic was restored with a circular synthesised beam size (FWHM) of $\theta_{\text {maj }}=\theta_{\text {min }}=0.75$, where $\theta_{\text {maj }}$ and $\theta_{\min }$ are the major and minor axes of the beam. The final $1 \sigma$ root mean square (rms) noise level in our maps is typically about $2.3 \mu \mathrm{Jy}$ beam $^{-1}$.

To quantify the effect of bandwidth smearing (BWS) in our $3 \mathrm{GHz}$ mosaic, we examined the behaviour of the ratio of the total integrated source flux density to its peak surface brightness as a function of the $\mathrm{S} / \mathrm{N}$ (e.g. Bondi et al. 2008; Novak et al. 2015; Smolčić et al., in prep.). This comparison showed that the effect of BWS in the full $3 \mathrm{GHz}$ mosaic of COSMOS is only up to $\sim 3 \%$, and no correction for BWS in the peak surface brightness is applied in the present study. To further examine the importance of BWS, we created images of a subsample of our sources from separate pointings where the source distance from the (nearest) phase centre is different. In addition to depending on the fractional bandwidth, the magnitude of BWS is directly proportional to the angular distance of the source from the phase centre. However, no significant radial smearing was seen in the aforementioned images, which lends further support to negligible BWS.

\section{3. $3 \mathrm{GHz}$ images and counterpart identification of the AzTEC SMGs}

The $3 \mathrm{GHz}$ images towards our SMGs are shown in Fig. 2. We note that at the redshifts of our sources, $z=0.834-5.298$, we are probing rest-frame frequencies of $v_{\text {rest }} \simeq 5.5-19 \mathrm{GHz}$ $\left(\lambda_{\text {rest }} \simeq 1.6-5.5 \mathrm{~cm}\right)$, which are dominated by non-thermal synchrotron radiation with the fraction of thermal emission becoming increasingly important at higher frequencies (e.g. Condon 1992; Murphy et al. 2012b). The $3 \mathrm{GHz}$ counterparts of our

\section{3 http://www.aips.nrao.edu/index.shtml}

4 CASA is developed by an international consortium of scientists based at the NRAO, the European Southern Observatory (ESO), the National Astronomical Observatory of Japan (NAOJ), the CSIRO Australia Telescope National Facility (CSIRO/ATNF), and the Netherlands Institute for Radio Astronomy (ASTRON) under the guidance of NRAO. See http://casa.nrao.edu 

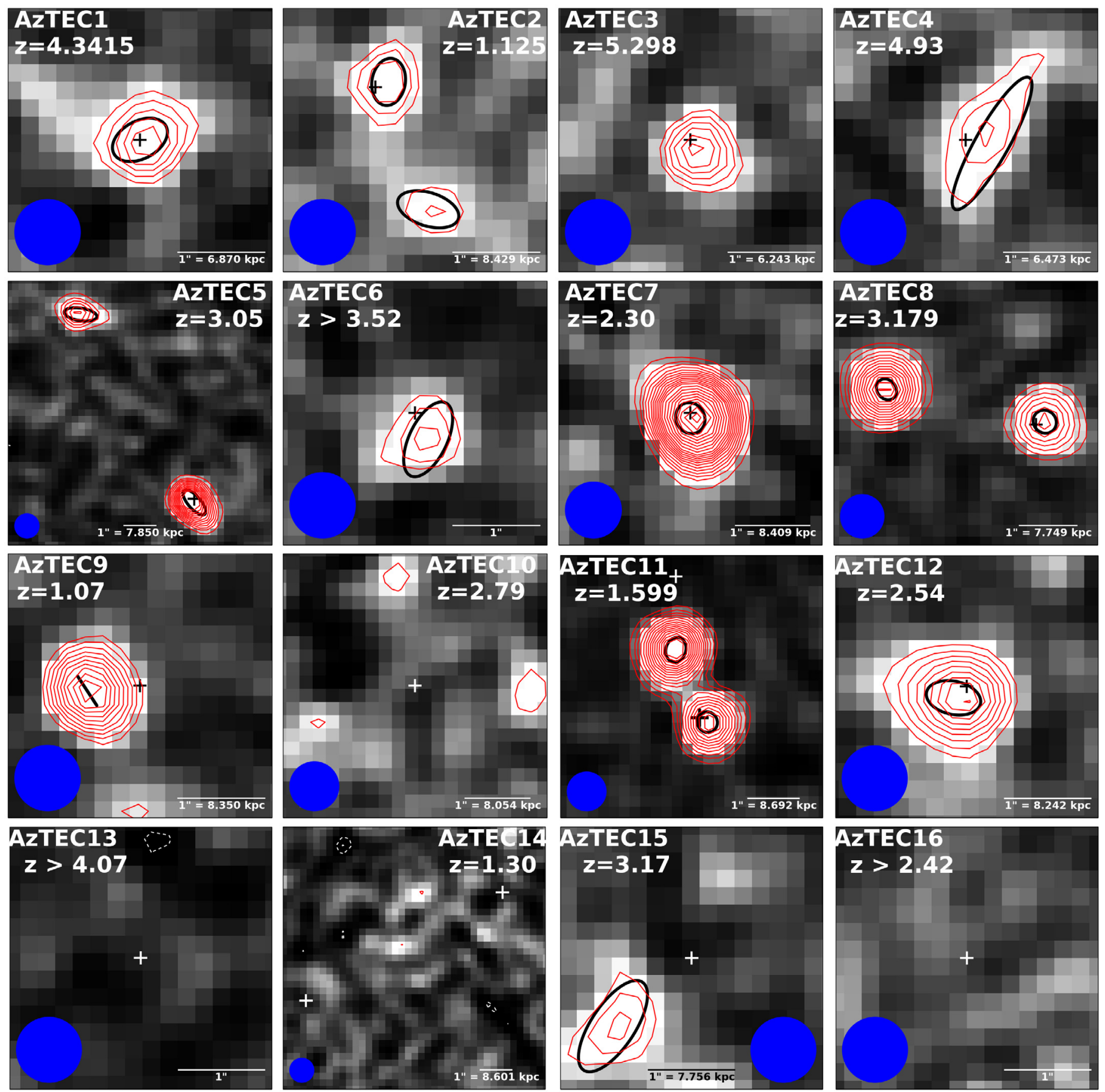

Fig. 2. VLA $3 \mathrm{GHz}$ images towards AzTEC1-30 displayed with north up and east left. The greyscale images are shown with power-law scaling (except for AzTEC28, where arcsinh scaling is used to better illustrate the intensity scale; a power-law scaling would be completely black), and the overlaid red contours start from $3 \sigma$ and increase in steps of $1 \sigma$ except for AzTEC5, 7, 8, 11, 12, 22, and 24 where the step is $\sqrt{2} \times \sigma$. The white dashed contours show the corresponding negative features (starting from $-3 \sigma$ ). The plus sign in each panel marks the (sub)mm peak position (SMA $890 \mu \mathrm{m}$ for AzTEC1-15: PdBI $1.3 \mathrm{~mm}$ for AzTEC16-30). The black thick ellipse shows the resulting Gaussian fit to the source (centred at the peak position, FWHM size, and PA). For AzTEC2 (both components), 3, 4, 5 (both components), 7, 8 (both components), 11-N, 11-S, and 12 the size represents an upper limit (see Table 2). Moreover, for AzTEC4 the peak position was not well determined, and for AzTEC9, 17a, and 27 only the major axis FWHM could be determined by JMFIT. The blue filled circle shows the synthesised beam size (0!'75 FWHM). We note that the areal coverage of the images differs from each other for illustrative purposes; a scale bar indicating the 1" projected length is shown in each panel, annotated with the corresponding proper length [kpc] at the quoted SMG redshift (except when only a lower limit to $z$ is available).

SMGs were identified by eye inspection of the corresponding images. The SMGs AzTEC1-9, 11-N and 11-S, 12, 15, 17a, 19a, $21 \mathrm{a}, 24 \mathrm{~b}$, and 27 are found to be associated with a $3 \mathrm{GHz}$ source (with a median offset of 0.26 ; Table 2), i.e. 18/39 or $\sim 46 \%$ (with a Poisson error on counting statistics of $\pm 11 \%$ ) of our sources are
$3 \mathrm{GHz}$-emitting SMGs. The $S / N$ of our detected $3 \mathrm{GHz}$ sources are in the range of $S / N=4.2-37.4$, AzTEC7 being the most significant detection. We note that the detection $\mathrm{S} / \mathrm{N}$ at $1.1 \mathrm{~mm}$ of these $3 \mathrm{GHz}$-emitting SMGs was found to be in the range of $S / N_{1.1 \mathrm{~mm}}=4.0-8.3$ (Scott et al. 2008). To summarise, $18 \mathrm{SMGs}$ 
O. Miettinen et al.: Spatial extent of radio emission in the COSMOS SMGs
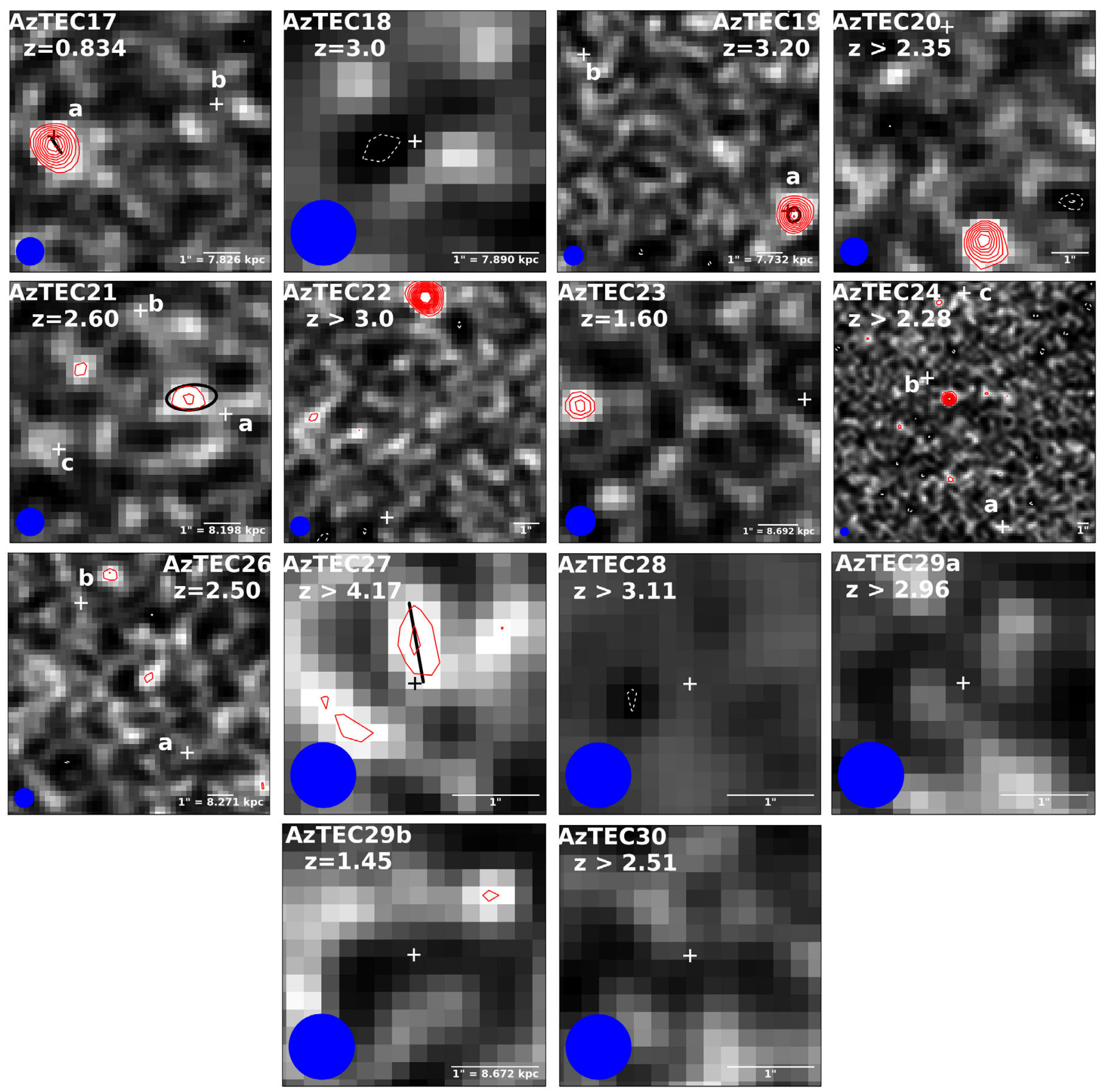

Fig. 2. continued.

in our sample are found to be associated with $3 \mathrm{GHz}$ emission. A selection of these SMGs, and the additional $3 \mathrm{GHz}$ radio sources not analysed further in the present study are discussed in more detail in Appendices A and B, respectively. The SMGs not detected at $3 \mathrm{GHz}$ are discussed in Appendix C.

\section{Analysis}

\subsection{Measuring the size of the radio-emitting region}

We used AIPS package to determine the deconvolved sizes of our $3 \mathrm{GHz}$ sources. Two-dimensional elliptical Gaussian fits to the image plane data were made using the AIPS task JMFIT. The fitting was performed inside a box containing the source, and the fit was restricted to the pixel values of $\geq 2.5 \sigma$. The results are given in Table 2, and illustrated in Fig. 2. To test the reliability of our size measurements, we simulated SMGs with an assigned size and varying $\mathrm{S} / \mathrm{N}$, and fit them in the same manner as the real sources. These simulations, described in Appendix D, suggest that the sizes provided by JMFIT are generally robust within the uncertainties assigned by the fitting task (see Fig. D.1, lower panel). As is often done in radio-continuum surveys, we considered a source to be resolved if its deconvolved FWHM size is larger than one-half the synthesised beam FWHM (e.g. Mundell et al. 2000; Ho \& Ulvestad 2001; Urquhart et al. 2009). AzTEC1, $6,15,19 \mathrm{a}, 21 \mathrm{a}$, and $24 \mathrm{~b}$ are resolved in both $\theta_{\text {maj }}$ and $\theta_{\text {min }}$, while 
Table 2. Results of Gaussian fits to the $3 \mathrm{GHz}$ sources.

\begin{tabular}{|c|c|c|c|c|c|c|c|c|c|}
\hline Source ID & $\begin{array}{l}\alpha_{2000.0}^{a} \\
{[\mathrm{~h}: \mathrm{m}: \mathrm{s}]} \\
\end{array}$ & $\begin{array}{r}\delta_{2000.0}{ }^{a} \\
{\left[0::^{\prime \prime}\right]} \\
\end{array}$ & $\begin{array}{c}I_{3 \mathrm{GHz}}{ }^{b} \\
{\left[\mu \mathrm{Jy} \mathrm{beam}^{-1}\right]}\end{array}$ & $\begin{array}{c}S_{3 \mathrm{GHz}}{ }^{b} \\
{[\mu \mathrm{Jy}]}\end{array}$ & $S / N$ & {$\left[{ }^{\prime \prime}\right] \quad F W H N$} & {$[\mathrm{kpc}]$} & $\begin{array}{r}\mathrm{PA}^{c} \\
{\left[{ }^{\circ}\right]} \\
\end{array}$ & $\begin{array}{c}\text { Offset } \\
{\left[{ }^{\prime \prime}\right]}\end{array}$ \\
\hline AzTEC1 & $095942.86( \pm 0.003)$ & $+022938.20( \pm 0.05)$ & $18.3 \pm 2.4$ & $28.3 \pm 5.2$ & 8.0 & $0.67_{-0.20}^{+0.17} \times 0.43_{-0.30}^{+0.19}$ & $4.6_{-1.4}^{+1.2} \times 3.0_{-2.1}^{+1.3}$ & $118.1_{-31.8}^{+31.8}$ & 0 \\
\hline \multirow[t]{2}{*}{$\mathrm{AzTEC} 2^{d}$} & $100008.04( \pm 0.003)$ & $+022612.26( \pm 0.06)$ & $15.0 \pm 2.4$ & $18.9 \pm 4.7$ & 6.0 & $0.54_{-0.21}^{+0.22} \times<0.38$ & $4.6_{-1.8}^{+1.8} \times<3.2$ & $170.9_{-32.7}^{+32.6}$ & 0.16 \\
\hline & $100008.01( \pm 0.007)$ & $+022610.81( \pm 0.08)$ & $10.0 \pm 2.4$ & $14.0 \pm 5.0$ & 4.3 & $0.72_{-0.44}^{+0.31} \times<0.38$ & $\ldots$ & $72.6_{-28.8}^{+28.8}$ & 1.51 \\
\hline AzTEC 3 & $100020.69( \pm 0.003)$ & $+023520.37( \pm 0.04)$ & $19.6 \pm 2.3$ & $19.6 \pm 2.3$ & 8.5 & $<0.38$ & $<2.4$ & $\ldots$ & 0.20 \\
\hline AzTEC4 & $095931.70( \pm 0.06)$ & $+023043.96( \pm 0.14)$ & $11.4 \pm 2.3$ & $31.5 \pm 7.7$ & 5.3 & $1.72_{-0.39}^{+0.37} x<0.38$ & $11.1_{-2.5}^{+2.4} \times<2.5$ & $150.6_{-8.3}^{+8.3}$ & 0.31 \\
\hline $\mathrm{AzTEC}^{d}$ & $100019.98( \pm 0.003)$ & $+023209.98( \pm 0.03)$ & $24.0 \pm 2.4$ & $42.8 \pm 5.9$ & 10.7 & $1.00_{-0.16}^{+0.14} \times<0.38$ & $8.7_{-1.4}^{+1.2} \times<3.3$ & $81.3_{-8.4}^{+8.4}$ & 6.56 \\
\hline AzTEC6 $^{e}$ & $100006.49( \pm 0.005)$ & $+023837.40( \pm 0.09)$ & $12.3 \pm 2.3$ & $22.4 \pm 5.8$ & 5.4 & $0.92_{-0.30}^{+0.26} \times 0.43_{-0.43}^{+0.26}$ & $\left(6.9_{-2.3}^{+1.9} \times 3.2_{-3.2}^{+2.0}\right)$ & $154.3_{-22.6}^{+22.5}$ & 0.34 \\
\hline AzTEC7 & $100018.06( \pm 0.001)$ & $+024830.43( \pm 0.01)$ & $89.5 \pm 2.4$ & $98.4 \pm 4.4$ & 37.4 & $0.42_{-0.05}^{+0.04} \times<0.38$ & $3.5_{-0.4}^{+0.4} \times<3.2$ & $29.8_{-6.2}^{+6.1}$ & 0.07 \\
\hline \multirow[t]{2}{*}{ AzTEC $8^{d}$} & $095959.33( \pm 0.001)$ & $+023441.05( \pm 0.02)$ & $38.8 \pm 2.4$ & $49.4 \pm 4.8$ & 16.3 & $0.41_{-0.17}^{+0.10} \times<0.38$ & $3.2_{-1.3}^{+0.8} \times<2.9$ & $46.4_{-0}^{+0}$ & 0.16 \\
\hline & $095959.51( \pm 0.001)$ & $+023441.60( \pm 0.01)$ & $73.5 \pm 2.4$ & $73.5 \pm 2.4$ & 29.4 & $<0.38$ & $<3.2$ & $\ldots$ & 2.62 \\
\hline AzTEC $9^{f}$ & $095957.29( \pm 0.002)$ & $+022730.54( \pm 0.03)$ & $29.4 \pm 2.2$ & $33.3 \pm 4.3$ & 13.0 & $\theta_{\text {maj }}=0.40_{-0.17}^{+0.13}$ & $\theta_{\text {maj }}=3.3_{-1.4}^{+1.1}$ & $33.2_{-24.1}^{+24.1}$ & 0.60 \\
\hline AzTEC11-N & $100008.90( \pm 0.001)$ & $+024009.52( \pm 0.01)$ & $57.0 \pm 2.3$ & $67.5 \pm 4.6$ & 24.4 & $0.40_{-0.08}^{+0.07} \times<0.38$ & $3.5_{-0.7}^{+0.6} \times<3.3$ & $31.3_{-20.5}^{+20.5}$ & 0.17 \\
\hline AzTEC12 & $100035.30( \pm 0.002)$ & $+024353.27( \pm 0.02)$ & $36.5 \pm 2.5$ & $52.5 \pm 5.2$ & 14.4 & $0.63_{-0.10}^{+0.10} \times<0.38$ & $5.2_{-0.8}^{+0.8} \times<3.1$ & $78.1_{-14.8}^{+14.8}$ & 0.20 \\
\hline AzTEC15 & $100012.95( \pm 0.006)$ & $+023434.92( \pm 0.10)$ & $12.2 \pm 2.4$ & $27.9 \pm 6.9$ & 5.4 & $1.21_{-0.32}^{+0.29} \times 0.50_{-0.50}^{+0.26}$ & $9.4_{-2.5}^{+2.2} \times 3.9_{-3.9}^{+2.0}$ & $146.3_{-15.5}^{+31.0}$ & 1.19 \\
\hline AzTEC $17 \mathrm{a}^{f}$ & $095939.19( \pm 0.001)$ & $+023403.58( \pm 0.02)$ & $34.9 \pm 2.3$ & $40.8 \pm 4.4$ & 15.1 & $\theta_{\text {maj }}=0.45_{-0.12}^{+0.10}$ & $\theta_{\text {maj }}=3.5_{-0.9}^{+0.8}$ & $34.4_{-17.1}^{+17.1}$ & 0.26 \\
\hline AzTEC19a & $100028.72( \pm 0.002)$ & $+023203.68( \pm 0.03)$ & $31.6 \pm 2.2$ & $45.3 \pm 5.0$ & 14.7 & $0.54_{-0.14}^{+0.11} \times 0.44_{-0.14}^{+0.21}$ & $4.2_{-1.1}^{+0.8} \times 3.4_{-1.1}^{+1.6}$ & $174.3_{-44.3}^{+44.2}$ & 0.27 \\
\hline AzTEC21a & $100002.63( \pm 0.01)$ & $+024642.14( \pm 0.10)$ & $9.4 \pm 2.3$ & $25.7 \pm 7.9$ & 4.2 & $1.34_{-0.44}^{+0.40} \times 0.67_{-0.50}^{+0.32}$ & $11.0_{-3.6}^{+3.3} \times 5.5_{-4.1}^{+2.6}$ & $95.2_{-21.8}^{+21.8}$ & 1.15 \\
\hline AzTEC $24 \mathrm{~b}^{e}$ & $100039.28( \pm 0.002)$ & $+023845.14( \pm 0.03)$ & $28.0 \pm 2.2$ & $37.6 \pm 4.7$ & 12.6 & $0.45_{-0.19}^{+0.13} \times 0.43_{-0.17}^{+0.15}$ & $\left(3.8_{-1.6}^{+1.1} \times 3.6_{-1.4}^{+1.3}\right)$ & $61.3_{-0}^{+0}$ & 2.67 \\
\hline $\mathrm{AzTEC} 27^{e, f}$ & $100039.21( \pm 0.004)$ & $+024052.65( \pm 0.12)$ & $9.9 \pm 2.3$ & $12.6 \pm 4.8$ & 4.3 & $\theta_{\text {maj }}=0.92_{-0.41}^{+0.34}$ & $\left(\theta_{\text {maj }}=6.4_{-2.8}^{+2.4}\right)$ & $10.1_{-13.1}^{+13.0}$ & 0.47 \\
\hline
\end{tabular}

Notes. The meaning of columns is as follows: (1): SMG name; (2) and (3): peak position of the fitted Gaussian; (4): peak surface brightness; (5): total flux density provided by the Gaussian fit; (6): $\mathrm{S} / \mathrm{N}$ as determined from the maximum pixel value with respect to the rms map noise; (7) and (8): deconvolved FWHM size $\left(\theta_{\text {maj }} \times \theta_{\text {min }}\right)$ in arcsec and physical kpc; (9) position angle of the fitted Gaussian measured from north through east; (10): projected angular offset from the (sub)mm position. ${ }^{(a)}$ Formal $1 \sigma$ uncertainties in seconds for $\alpha_{2000.0}$ and arcseconds for $\delta_{2000.0}$ returned by JMFIT are given in parentheses. ${ }^{(b)}$ The quoted error in $I_{3 \mathrm{GHz}}$ is the $1 \sigma \mathrm{rms}$ noise in the map determined inside a $\sim 300 \square^{\prime \prime}$ box placed near the SMG, and which did not include any $3 \mathrm{GHz}$ sources. The uncertainty in $S_{3 \mathrm{GHz}}$ represents the formal error determined with JMFIT. The uncertainties do not include the absolute calibration uncertainty. ${ }^{(c)}$ The size and PA uncertainties represent the minimum/maximum values as returned by JMFIT. We note that the PA is formally defined to range from $0^{\circ}$ to $180^{\circ}$, but for example for AzTEC 2 the maximum PA value is 203.5 , which is equivalent to an angle of $203^{\circ} .5-180^{\circ}=23^{\circ} .5$. The minimum and maximum PA values for AzTEC 8 and $24 \mathrm{~b}$ are equal to the nominal value, and hence the quoted uncertainties are equal to zero. ${ }^{(d)}$ Two $3 \mathrm{GHz}$ sources were detected. No linear size is reported for the secondary component towards AzTEC2 because of its unknown redshift. ${ }^{(e)}$ For AzTEC6, 24b, and 27 only a lower redshift limit is available (see Table 1), and the linear FWHM size quoted in parentheses was calculated at that lower $z$ limit; these linear sizes were not included in the statistical size analysis. ${ }^{(f)}$ For AzTEC9, 17a, and 27 only the major axis could be determined by JMFIT (minor axis $=0$ ).

AzTEC2 (both components), 4, 5 (both components), 7, 8, 11-N, $11-\mathrm{S}$, and 12 are resolved in $\theta_{\text {maj }}$ but unresolved in $\theta_{\min }$. AzTEC3 and the additional component towards AzTEC8 are unresolved in both axes. The upper size limit for unresolved sources was set to one-half the synthesised beam FWHM $(<0$ '.38). For AzTEC9, $17 \mathrm{a}$, and 27 only the major axis FWHM could be determined by JMFIT, while the fitting task did not provide a value for the minor axis FWHM (the output value $=0$ ). In Col. 8 in Table 2, we give the projected linear FWHM size for those SMGs with known redshift (i.e. not just a lower limit to $z$ derived using the Carilli \& Yun $(1999,2000)$ method). As mentioned earlier in Sect. 2.2, no correction for the negligible BWS in the peak surface brightness or FWHM size was applied.

To calculate the statistical properties of our radio-emission size distribution, we applied survival analysis to take the upper limits to the size into account. We assumed that the censored data follow the same distribution as the uncensored values, and we used the Kaplan-Meier (K-M) method to construct a model of the input data (for this purpose, we used the Nondetects And Data Analysis for environmental data (NADA; Helsel 2005) package for R). However, because more than $50 \%$ of our minor axis data are censored, the K-M estimator could not be used to determine the median value of the minor axis length, and hence
Table 3. $3 \mathrm{GHz}$ size distribution statistics.

\begin{tabular}{|c|c|}
\hline Parameter & Value $^{a}$ \\
\hline Mean $\theta_{\text {maj }}$ & $0.71 \pm 0.09(5.5 \pm 0.7 \mathrm{kpc})$ \\
\hline Median $\theta_{\text {maj }}$ & $0^{\prime} .54 \pm 0.11(4.2 \pm 0.9 \mathrm{kpc})$ \\
\hline Mean $\theta_{\min }$ & $0.45 \pm 0.02(3.3 \pm 0.2 \mathrm{kpc})$ \\
\hline Median $\theta_{\min }{ }^{b}$ & $0.35 \pm 0.04(2.3 \pm 0.4 \mathrm{kpc})$ \\
\hline Standard deviation of $\theta_{\text {maj }}$ & $0.39(2.9 \mathrm{kpc})$ \\
\hline Standard deviation of $\theta_{\min }$ & $00^{\prime} 07(0.8 \mathrm{kpc})$ \\
\hline $95 \%$ confidence interval of $\theta_{\text {maa }}^{c}$ & $0.54-0.99(4.0-6.9 \mathrm{kpc})$ \\
\hline $95 \%$ confidence interval of $\theta_{\min }{ }^{c}$ & $0.42-0.49(2.9-3.7 \mathrm{kpc})$ \\
\hline
\end{tabular}

Notes. ${ }^{(a)}$ The sample size of the major (minor) axis angular sizes is 18 (15), while that of the linear sizes is 15 (13), i.e. the number of SMGs with either a $z_{\text {spec }}$ or $z_{\text {phot }}$ value available. ${ }^{(b)}$ The median value of $\theta_{\min }$ could not be derived using the K-M estimator. Hence, it was calculated using the MLE (assuming lognormal distribution), which is almost identical to the K-M function. (c) A two-sided 95\% confidence interval for the mean value computed using the K-M method.

its value was derived using the Maximum Likelihood Estimator (MLE) of the survivor function. The mean, median, standard deviation, and $95 \%$ confidence interval of the deconvolved FWHM sizes are given in Table 3. For example, the median value of 

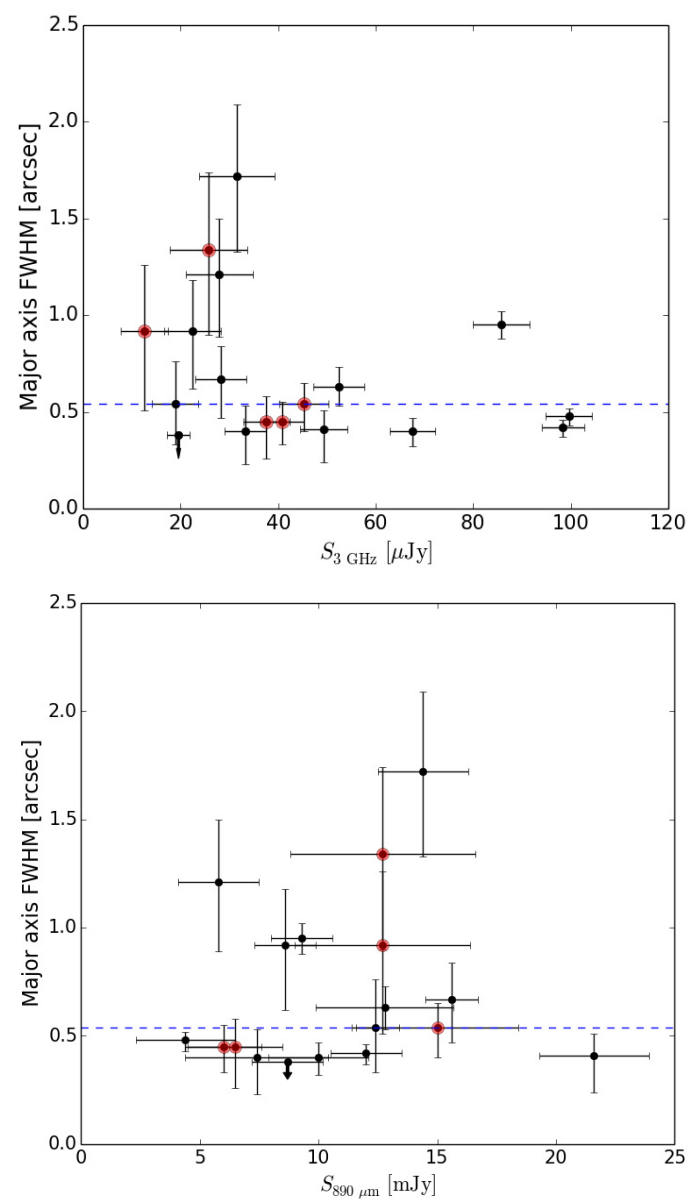

Fig. 3. Top: angular FWHM of the major axis at $3 \mathrm{GHz}$ as a function of the $3 \mathrm{GHz}$ flux density. Bottom: same as above but as a function of the $890 \mu \mathrm{m}$ flux density. For AzTEC17a, 19a, 21a, 24b, and 27 the value of $S_{890 \mu \mathrm{m}}$ was calculated from $S_{1.3 \mathrm{~mm}}$ by assuming that the dust emissivity index is $\beta=1.5$; these data points are highlighted by red filled circles in both panels. The horizontal dashed line marks the median major axis FWHM of 0.'54. The upper size limit for AzTEC3 is indicated by a downward pointing arrow.

the deconvolved $\theta_{\text {maj }}$ among the $18 \mathrm{SMGs}$ detected at $3 \mathrm{GHz}$ is $0 . ' 54 \pm 0 . ' 11$ (FWHM), and the median major axis FWHM in linear units is $4.2 \pm 0.9 \mathrm{kpc}$ as estimated for our SMGs with known redshift (15 sources with either a $z_{\text {spec }}$ or $z_{\text {phot }}$ value available). In the subsequent size analysis we will employ the deconvolved FWHM of the major axis because the value of $\theta_{\text {maj }}$ would set the physical extent of a disk-like galaxy, while the minor axis, assuming this simplified disk-like geometry, would be given by $\theta_{\text {min }}=\theta_{\text {maj }} \times \cos (i)$ (defined so that for a disk viewed face-on $\left.\left(i=0^{\circ}\right), \theta_{\min }=\theta_{\text {maj }}\right)$.

In Fig. 3, we plot the deconvolved major axis FWHM sizes as a function of the $3 \mathrm{GHz}$ flux density (upper panel) and $890 \mu \mathrm{m}$ flux density (lower panel), where $S_{890 \mu \mathrm{m}}$ for AzTEC1-15 was taken from Younger et al. (2007, 2009), and for AzTEC17a, 19a, 21a, 24b, and 27 the value of $S_{890 \mu \mathrm{m}}$ was calculated from the $1.3 \mathrm{~mm}$ flux density by assuming that $\beta=1.5$ (Miettinen et al. 2015). No statistically significant correlation can be seen between the $3 \mathrm{GHz}$ size and the radio or submm flux density, but we note that the largest angular major axis FWHM sizes are preferentially found among the sources with the lowest $3 \mathrm{GHz}$ flux densities, although the size uncertainties for those sources are the highest.
Table 4. Radio continuum characteristics of the $3 \mathrm{GHz}$ detections.

\begin{tabular}{cccc}
\hline \hline Source ID & $\begin{array}{c}S_{1.4 \mathrm{GHz}}{ }^{a} \\
{[\mu \mathrm{Jy}]}\end{array}$ & $\begin{array}{c}T_{\mathrm{B}}{ }^{b} \\
{[\mathrm{~K}]}\end{array}$ & $\alpha_{1.4 \mathrm{GHz}}^{3 \mathrm{GHz}}$ \\
\hline AzTEC1 & $48 \pm 12$ & $8.9 \pm 5.0$ & $-(0.69 \pm 0.61)$ \\
AzTEC2 & $76 \pm 14$ & $8.8 \pm 7.4$ & $-(1.83 \pm 0.77)$ \\
AzTEC3 & $<30$ & $>16.3$ & $>-0.91$ \\
AzTEC4 & $<36$ & $1.4 \pm 0.7$ & $>-0.91$ \\
AzTEC5 & $126 \pm 15$ & $12.9 \pm 2.1$ & $-(0.50 \pm 0.24)$ \\
AzTEC5-N & $85 \pm 15$ & $5.8 \pm 1.9$ & $-(0.90 \pm 0.45)$ \\
AzTEC6 & $<38$ & $3.6 \pm 2.4$ & $>-1.48$ \\
AzTEC7 & $132 \pm 22$ & $75.9 \pm 16.6$ & $-(0.39 \pm 0.21)$ \\
AzTEC8-W & $102 \pm 13$ & $40.0 \pm 26.6$ & $-(0.95 \pm 0.32)$ \\
AzTEC8-E & $160 \pm 23$ & $>67$ & $-(1.02 \pm 0.17)$ \\
AzTEC9 & $68 \pm 13$ & $25.0 \pm 18.8$ & $-(1.10 \pm 0.30)$ \\
AzTEC11-N & $138 \pm 26$ & $57.4 \pm 21.9$ & $-(0.94 \pm 0.30)$ \\
AzTEC11-S & $132 \pm 26$ & $58.8 \pm 11.4$ & $-(0.37 \pm 0.27)$ \\
AzTEC12 & $98 \pm 16$ & $18.0 \pm 6.0$ & $-(0.82 \pm 0.34)$ \\
AzTEC15 & $<32$ & $2.6 \pm 1.5$ & $>-0.93$ \\
AzTEC17a & $68 \pm 13$ & $23.4 \pm 11.6$ & $-(0.88 \pm 0.28)$ \\
AzTEC19a & $78 \pm 12$ & $21.1 \pm 10.1$ & $-(0.71 \pm 0.37)$ \\
AzTEC21a & $<44$ & $1.9 \pm 1.4$ & $>-1.63$ \\
AzTEC24b & $63 \pm 13$ & $25.3 \pm 18.2$ & $-(0.68 \pm 0.43)$ \\
AzTEC27 & $<39$ & $2.0 \pm 1.8$ & $>-2.63$ \\
\hline
\end{tabular}

Notes. ${ }^{(a)}$ The values of $S_{1.4 \mathrm{GHz}}$ were taken from the COSMOS VLA Deep Catalogue May 2010 (Schinnerer et al. 2010) except for AzTEC1, 8 , and 11 . AzTEC1 exhibits $4 \sigma 1.4 \mathrm{GHz}$ emission, hence is not listed in the VLA catalogue, which is comprised of $\geq 5 \sigma$ sources. The value of $S_{1.4 \mathrm{GHz}}$ for AzTEC1 was taken as the peak surface brightness multiplied by 1.15 to correct for BWS (Younger et al. 2007). The resulting flux density of $48 \pm 12 \mu \mathrm{Jy}$ agrees with the value reported by Younger et al. (2007, Table 2 therein). For AzTEC8 and 11 we adopted the 1.4 GHz flux densities from Younger et al. (2009, Table 2 therein), and multiplied them by 1.15 to correct for BWS as noted by the authors. The COSMOS VLA catalogue values of $S_{1.4 \mathrm{GHz}}$ for AzTEC8-W and AzTEC8-E are $237 \pm 52 \mu \mathrm{Jy}$ and $186 \mu \mathrm{Jy}$ (no error given), respectively. However, AzTEC8-E is a stronger $1.4 \mathrm{GHz}$ source than AzTEC8-W (Younger et al. 2009). For AzTEC11 the COSMOS VLA catalogue gives an integrated flux density value of $S_{1.4 \mathrm{GHz}}=302 \pm 45 \mu \mathrm{Jy}$. Hence, we adopted the $S_{1.4 \mathrm{GHz}}$ values for AzTEC11-N and 11-S from Younger et al. (2009). The $3 \sigma$ upper limits are reported for the nondetections, where $1 \sigma \simeq 10-14.7 \mu \mathrm{Jy}_{\text {beam }}{ }^{-1}$. ${ }^{(b)}$ The value of $T_{\mathrm{B}}$ refers to the Rayleigh-Jeans brightness temperature at $v_{\mathrm{obs}}=3 \mathrm{GHz} .{ }^{(c)}$ Radio spectral index between the observed-frame frequencies of $1.4 \mathrm{GHz}$ and $3 \mathrm{GHz}$.

\subsection{Spectral index between 1.4 and $3 \mathrm{GHz}$, and $3 \mathrm{GHz}$ brightness temperature}

To further characterise the radio continuum properties of our SMGs, we derived their radio spectral index between 1.4 and $3 \mathrm{GHz}\left(\alpha_{1.4 \mathrm{GHz}}^{3 \mathrm{GHz}}\right)$, and the observed-frame $3 \mathrm{GHz}$ brightness temperature $\left(T_{\mathrm{B}}\right)$. The $1.4 \mathrm{GHz}$ flux densities were taken from the COSMOS VLA Deep Catalogue (Schinnerer et al. 2010) for all the sources except AzTEC1, 8, and 11 for which $S_{1.4 \mathrm{GHz}}$ was taken/revised from Younger et al. (2007, 2009); see Col. (2) in Table 4. The angular resolution in the $1.4 \mathrm{GHz}$ VLA Deep mosaic is 2.'5 (Schinnerer et al. 2010), while that of the $1.4 \mathrm{GHz}$ VLA-COSMOS Large Project data, used by Younger et al. (2007, 2009), is $11^{\prime \prime} 5 \times 11^{\prime \prime} 4$ (Schinnerer et al. 2007). These are about 3.3 and 1.9 times poorer than in our $3 \mathrm{GHz}$ mosaic, respectively. This difference was not taken into account, but we used the $1.4 \mathrm{GHz}$ peak surface brightness as the corresponding source flux density, except for AzTEC8 and 11, for which Gaussianfit based flux densities from Younger et al. (2009) were used 
(see Table 4). The 1.4 and $3 \mathrm{GHz}$ flux densities were then used to derive $\alpha_{1.4 \mathrm{GHz}}^{3 \mathrm{GHz}}$, where we define the spectral index as $S_{v} \propto v^{\alpha}$. The derived spectral indices are listed in Col. (4) in Table 4; the quoted errors were propagated from those of the flux densities. The $3 \mathrm{GHz}$ Rayleigh-Jeans brightness temperature was calculated as $T_{\mathrm{B}}=c^{2} S_{v} /\left(2 k_{\mathrm{B}} v^{2} \Omega\right)$, where $c$ is the speed of light, $k_{\mathrm{B}}$ is the Boltzmann constant, and the solid angle subtended by the Gaussian source was derived from $\Omega=\pi \theta_{\mathrm{maj}}^{2} /(4 \ln 2)$. The uncertainties in $T_{\mathrm{B}}$ were derived from those associated with $S_{3 \mathrm{GHz}}$ and the $3 \mathrm{GHz}$ major axis FWHM size (see Col. (3) in Table 4). We note that Smolčić et al. (2015a) already derived the values of $\alpha_{1.4 \mathrm{GHz}}^{3 \mathrm{GHz}}$ for AzTEC1 $(-0.90 \pm 0.46)$ and AzTEC3 $(>-0.09)$. Given the large associated uncertainties, the present spectral index for AzTEC1 $(-0.69 \pm 0.61)$ is consistent with the previous value, while the lower limit of $\alpha_{1.4 \mathrm{GHz}}^{3 \mathrm{GHz}}>-0.91$ we have derived for AzTEC3 is different because of the lower $3 \mathrm{GHz}$ flux density determined here.

In the top panel of Fig. 4, we plot the $3 \mathrm{GHz}$ angular major axis FWHM sizes as a function of $\alpha_{1.4 \mathrm{GHz}}^{3 \mathrm{GHz}}$, while the bottom panel shows the $3 \mathrm{GHz} T_{\mathrm{B}}$ values as a function of $\alpha_{1.4 \mathrm{GHz}}^{3 \mathrm{GHz}}$. Among local luminous and ultraluminous infrared galaxies or (U)LIRGs, it has been found that smaller sources exhibit a flatter radio spectral index (Condon et al. 1991; Murphy et al. 2013). The observed trend of more compact sources exhibiting flatter radio spectral indices is an indication of increased free-free absorption by ionised gas (i.e. free electrons gain energy by absorbing radio photons during their collisions with ions). No such correlation is obvious in our data, and the lower $\alpha_{1.4 \mathrm{GHz}}^{3 \mathrm{GHz}}$ limits muddy the interpretation. Also, no obvious trend is found between $T_{\mathrm{B}}$ and $\alpha_{1.4 \mathrm{GHz}}^{3 \mathrm{GHz}}$ as shown in the bottom panel of Fig. 4 , i.e. sources with a higher $T_{\mathrm{B}}$ do not appear to show spectral flattening (cf. Fig. 1 in Murphy et al. 2013; we note their different definition of $S_{v} \propto v^{-\alpha}$ ). The low values of $T_{\mathrm{B}}$, ranging from $1.4 \pm 0.7 \mathrm{~K}$ to $75.9 \pm 16.6 \mathrm{~K}$, show that the observed $3 \mathrm{GHz}$ radio emission from our SMGs is powered by star formation activity and no evidence of buried AGN activity is visible in our data (AGN have $T_{\mathrm{B}} \gtrsim 3.2 \times 10^{4} \mathrm{~K}$ at $8.44 \mathrm{GHz}\left(=v_{\text {rest }}\right.$ at $z \simeq 1.8$ for $v_{\mathrm{obs}}=3 \mathrm{GHz}$ ); Condon et al. 1991; Murphy et al. 2013).

\section{Comparison with literature}

\subsection{Previous size measurements of the COSMOS/AzTEC SMGs}

In addition to the present work, the sizes of AzTEC1, 3, 4, 5, and 8 have been previously determined at $3 \mathrm{GHz}$ and/or other observed frequencies (see Table 5). Below, we discuss the size measurements of these five high-redshift SMGs in more detail.

AzTEC1. We have found that AzTEC1 is resolved $\left(0 .{ }^{\prime} 67_{-0.20}^{+0.17} \times 0.0^{\prime} 43_{-0.30}^{+0.19}\right)$ in our $3 \mathrm{GHz}$ image. Smolčić et al. (2015a) employed a $3 \mathrm{GHz}$ submosaic of the COSMOS field, which was based on $130 \mathrm{~h}$ of observations, and had a $1 \sigma \mathrm{rms}$ noise level of $4.5 \mu \mathrm{Jy}$ beam $^{-1}$, i.e. about two times higher than in the data used here. AzTEC1 remained unresolved (upper size limit was set to $<00^{\prime} 7$ ) in the previous map although the angular resolution was slightly higher, i.e. $0.7 \times 0 . ' 6$. The $0.35 \times 0.25$ resolution SMA $890 \mu \mathrm{m}$ observations of AzTEC1 by Younger et al. (2008) showed the source size to be $\theta_{\text {maj }} \times \theta_{\text {min }} \sim 00^{\prime} 3 \times 0,0^{\prime} 2$ $(\sim 0.4 \times 0 . ! 3)$ when modelled as a Gaussian (elliptical disk). This Gaussian major axis FWHM at $\lambda_{\text {rest }}=167 \mu \mathrm{m}$ is $2.2 \pm 0.6$ times smaller than the size at $\lambda_{\text {rest }}=1.9 \mathrm{~cm}$ we have derived (see Fig. 5). Miettinen et al. (in prep.) used ALMA (PI: A. Karim) to observe AzTEC1 at $\lambda_{\text {obs }}=870 \mu \mathrm{m}\left(\lambda_{\text {rest }}=163 \mu \mathrm{m}\right)$ continuum and an angular resolution of $0.30 \times 0.29$. Fitting the
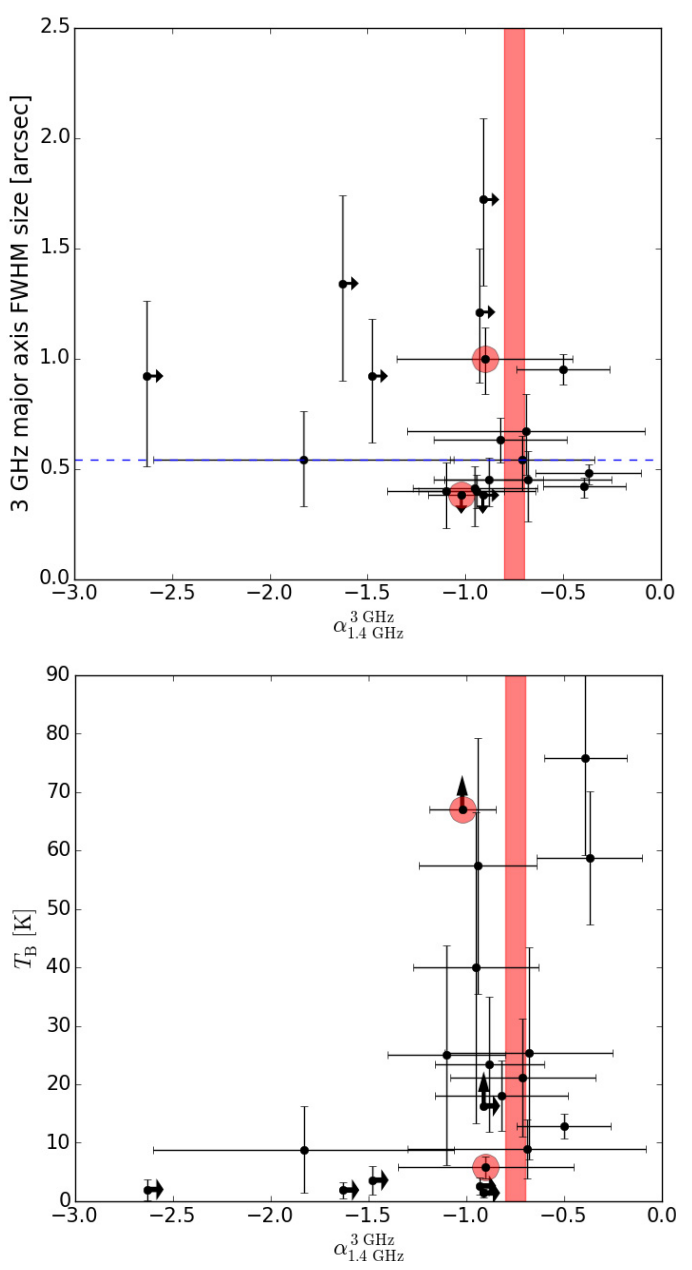

Fig. 4. Top: $3 \mathrm{GHz}$ angular major axis FWHM size plotted against the radio spectral index between the observed frequencies of 1.4 and $3 \mathrm{GHz}$. The arrows pointing right indicate lower limits to $\alpha_{1.4 \mathrm{GHz}}^{3 \mathrm{GH}}$, while the down-pointing arrows show the upper size limits. The data points highlighted with red filled circles are for the additional $3 \mathrm{GHz}$ components seen towards AzTEC5 (upper data point) and AzTEC8 (lower data point); as discussed in Appendix A, those are probably not physically related to the SMGs. The horizontal dashed line marks the median major axis FWHM size of our SMGs (0.'54). For reference, the red shaded region shows the radio spectral index range of $-0.8 \ldots-0.7$, which is typical of the non-thermal synchrotron radio emission from starforming galaxies. Within the errors, 11 out of 20 sources $(55 \% \pm 17 \%)$ shown here have a $\alpha_{1.4 \mathrm{GHz}}^{3 \mathrm{GHz}}$ value consistent with this range, and 6 additional sources have a lower limit to $\alpha_{1.4 \mathrm{GHz}}^{3 \mathrm{GHz}}$ less than -0.8 . Bottom: $3 \mathrm{GHz}$ brightness temperature as a function of $\alpha_{1.4 \mathrm{GHz}}^{3 \mathrm{GHz}}$. The symbols are as in the top panel, except that the arrows pointing up indicate the lower $T_{\mathrm{B}}$ limits, and the lower and upper red filled circles are for AzTEC5-N and AzTEC8-E, respectively.

source in the $870 \mu \mathrm{m}$ image plane using JMFIT results in the deconvolved FWHM size of $0^{\prime} .39_{-0.01}^{+0.01} \times 0.0^{\prime} 31_{-0.01}^{+0.01}$, which is fairly similar to the size derived by Younger et al. (2008) at a comparable wavelength. Based on deep UltraVISTA observations ( $\sim$ '. $^{\prime}$ resolution at FWHM), Toft et al. (2014) derived an upper limit of $<2.6 \mathrm{kpc}$ to the observed-frame NIR size of AzTEC1. The authors fit two-dimensional Sérsic models to the surface brightness distributions, and calculated the effective radius encompassing half the light of the model. This size scale corresponds to a Gaussian half width at half maximum (HWHM) size (see Table 1 in Toft et al. 2014), and to be compared with our FWHM diameters we multiplied the sizes from Toft et al. (2014) by 2 . 
O. Miettinen et al.: Spatial extent of radio emission in the COSMOS SMGs

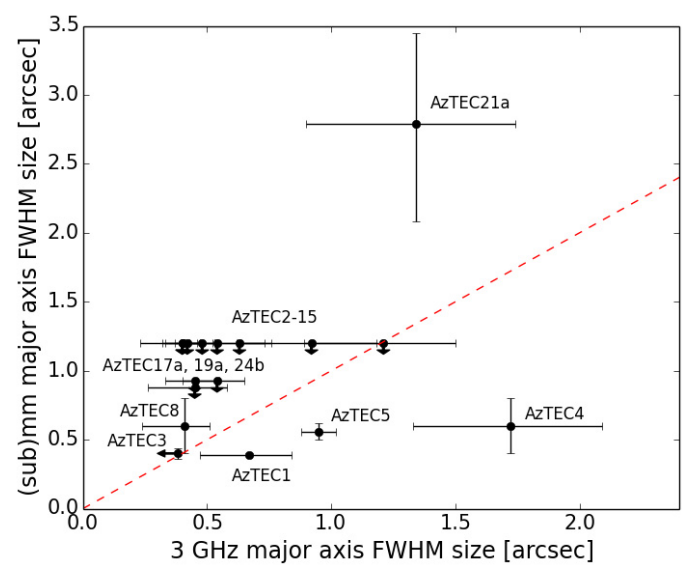

Fig. 5. (Sub)mm angular major axis FWHM sizes of our SMGs (at $890 \mu \mathrm{m}$ from Younger et al. 2007, 2008, 2009, at $870 \mu \mathrm{m}$ from Younger et al. 2010, or at $1.3 \mathrm{~mm}$ from Miettinen et al. 2015) plotted against their $3 \mathrm{GHz}$ major axis FWHM sizes. For AzTEC1, 3, 4, 5, 8, and 21a the (sub)mm emission has been (marginally) resolved. For AzTEC1, 4, and 8 we plot the Gaussian-fit size from Younger et al. $(2008,2010)$, while the size of AzTEC3 is that determined from ALMA observations at $1 \mathrm{~mm}$ by Riechers et al. (2014). For AzTEC5, the submm size was derived from the $994 \mu \mathrm{m}$ ALMA image (Miettinen et al., in prep.) using JMFIT (see text for details). Similarly, the plotted $1.3 \mathrm{~mm}$ size of AzTEC21a was derived by fitting the source using JMFIT for a better comparison with the present $3 \mathrm{GHz}$ size. The AzTEC27 data point is not shown due to its non-Gaussian shape at $1.3 \mathrm{~mm}$ (see text for details). Among AzTEC2-15, the upper $890 \mu \mathrm{m}$ size limits - marked with arrows pointing down - are $\lesssim 1^{\prime \prime}$. 2 (Younger et al. 2007, 2009), while the upper limits to the $1.3 \mathrm{~mm}$ sizes of AzTEC17a, 19a, and 24b are set to one-half the synthesised beam major axis size at FWHM (Miettinen et al. 2015; Table 2 therein). The red dashed line indicates where the radio and (sub)mm sizes are equal.

The physical radius reported by Toft et al. (2014) corresponds to a diameter of $<0$ '. 76 in angular units, which suggests that the rest-frame UV-optical size of AzTEC1 could be comparable to its FIR size $\left(\theta_{\text {maj }} \sim 0^{\prime \prime} 3\right)$ and/or $1.9 \mathrm{~cm}$ radio continuum size $\left(\theta_{\text {maj }}=0{ }^{\prime} 67_{-0.20}^{+0.17}\right)$.

AzTEC3. This source is unresolved in our $3 \mathrm{GHz}$ image, similarly to that found by Smolčić et al. (2015a) in their $3 \mathrm{GHz}$ image (upper size limit was set to $<00^{\prime} 7$ ). Riechers et al. (2014) used ALMA to observe AzTEC3 at an angular resolution of $0 .{ }^{\prime} 63 \times 0 . ' 56$. In the $\lambda_{\text {obs }}=1 \mathrm{~mm}\left(\lambda_{\text {rest }}=159 \mu \mathrm{m}\right)$ continuum, the deconvolved FWHM size of AzTEC3 was derived to be $0 .{ }^{\prime} 40_{-0.04}^{+0.04} \times 0{ }^{\prime} 17_{-0.17}^{+0.08}$, while in the $\lambda_{\text {rest }}=158 \mu \mathrm{m}$ [C II] line emission the size was found to be larger, $0.63_{-0.09}^{+0.09} \times 0 .{ }^{\prime} 34_{-0.15}^{+0.10}$. We have derived the $\lambda_{\text {rest }}=1.6 \mathrm{~cm}$ upper FWHM size limit of AzTEC3 to be $<0$ '. 38 , which is smaller than the aforementioned rest-frame FIR continuum and [C II] sizes (although the major axis FWHM at $\lambda_{\text {rest }}=159 \mu \mathrm{m}$ is marginally consistent with our upper size limit; see Fig. 5). The observed-frame NIR diameter of AzTEC3 derived by Toft et al. (2014) is $<4.8 \mathrm{kpc}$, i.e. $<0$ ' 76 , which is also consistent with our radio emission FWHM size, and with the FIR FWHM size from Riechers et al. (2014).

AzTEC4. For this source, the FWHM size at $3 \mathrm{GHz}$ is determined to be $11^{\prime \prime} 72_{-0.39}^{+0.37} \times<0$ '. 38 . The source appears elongated with a major-to-minor axis ratio of $>3.5$, but as shown in Fig. 2, the $3 \mathrm{GHz}$ peak position is not well determined by JMFIT. The 0 .' $86 \times 0$ '. 77 resolution SMA $870 \mu$ m observations of AzTEC4 by Younger et al. (2010) showed the source size to be $\theta_{\text {maj }} \times \theta_{\text {min }}=$ $\left(0 \prime^{\prime} 6 \pm 0.2\right) \times\left(0 \prime^{\prime} 4 \pm 0.0^{\prime} 2\right)\left(\left(1^{\prime \prime} .0 \pm 0.0^{\prime} 4\right) \times\left(0 \prime^{\prime} 7 \pm 0.0^{\prime} 6\right)\right)$ when modelled as a Gaussian (elliptical disk). This Gaussian major axis FWHM at $\lambda_{\text {rest }}=147 \mu \mathrm{m}$ is $2.9_{-1.2}^{+2.4}$ times smaller than the size at $\lambda_{\text {rest }}=1.7 \mathrm{~cm}$ we have derived (see Fig. 5). The observedframe NIR diameter of AzTEC4 derived by Toft et al. (2014) is $<5.0 \mathrm{kpc}(<0$ ! 78), which is consistent with the Gaussian FWHM at rest-frame FIR derived by Younger et al. (2010).

AzTEC5. The $3 \mathrm{GHz}$ FWHM size we have determined for this source is $0.95_{-0.07}^{+0.07} \times<0.38$, i.e. the major axis is resolved while the minor axis is unresolved. Miettinen et al. (in prep.) used ALMA (PI: A. Karim) to observe AzTEC5 at $\lambda_{\text {obs }}=994 \mu \mathrm{m}\left(\lambda_{\text {rest }} \simeq 245 \mu \mathrm{m}\right)$ continuum and an angular resolution of $0.52 \times 0.30$. The source was resolved into two components with a projected separation of $0.75(\sim 5.86 \mathrm{kpc}$ at the source redshift). The northern ALMA component is perfectly coincident with our $3 \mathrm{GHz}$ source ( $0{ }^{\prime}$.03 offset), but we note that the $3 \mathrm{GHz}$ emission extends towards the southern ALMA FIR component, and hence the detected $3 \mathrm{GHz}$ emission encompasses the two ALMA-detected components. Fitting the ALMA sources in the image plane using JMFIT results in the deconvolved FWHM size of 0 ' $^{\prime} 45_{-0.02}^{+0.04} \times 0 .{ }^{\prime} 28_{-0.08}^{+0.04}$ for the northern component, and $0.6_{-0.06}^{+0.06} \times 0.0^{\prime} 38_{-0.09}^{+0.08}$ for the southern component. The major axis FWHM length of $0 .^{\prime} 95_{-0.07}^{+0.07}$ at $3 \mathrm{GHz}$ is comparable to the sum of the major axes of the ALMA emission from the two sources $\left(\simeq 1^{\prime \prime}\right)$. Toft et al. (2014) used high resolution (FWHM $\sim 0$ !'2) data from the Wide Field Camera 3 (WFC3) aboard the Hubble Space Telescope to determine the rest-frame UV/optical size of AzTEC5. The diameter derived from their reported radius is $1.0 \pm 0.8 \mathrm{kpc}\left(0{ }^{\prime} 12 \pm 0\right.$ ' $\left.^{\prime} 10\right)$. The major axis FWHM of AzTEC5 at $3 \mathrm{GHz}$ is $7.9 \pm 6.6$ times larger than its $\mathrm{UV} /$ optical diameter.

AzTEC8. For this source, the FWHM size at $3 \mathrm{GHz}$ is determined to be 0 . $^{\prime} 41_{-0.17}^{+0.10} \times<0.38$. The major axis is only marginally resolved, while the minor axis is unresolved. The $0 . ' 86 \times 0$ 0.55 resolution SMA $870 \mu$ m observations of AzTEC8 by Younger et al. (2010) showed the source size to be $\theta_{\text {maj }} \times$

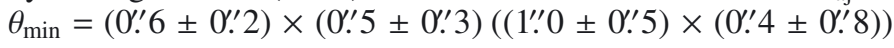
when modelled as a Gaussian (elliptical disk). This Gaussian major axis FWHM at $\lambda_{\text {rest }}=208 \mu \mathrm{m}$ is $1.5_{-0.7}^{+1.8}$ times larger than the radio size at $\lambda_{\text {rest }}=2.4 \mathrm{~cm}$ we have derived (see Fig. 5). The observed-frame NIR diameter of AzTEC8 derived by Toft et al. (2014), <6.0 kpc $(<0.78)$, is consistent with our $3 \mathrm{GHz}$ size and the Gaussian rest-frame FIR size determined by Younger et al. (2010).

For the remaining of our SMGs mostly upper size limits at other wavelengths are available. Younger et al. (2007, 2009) constrained the observed-frame $890 \mu \mathrm{m}$ sizes of AzTEC1-15 to $\lesssim 1^{\prime \prime}$. 2 (i.e. the sources were unresolved), with the exception of AzTEC11 that was found to be resolved but best modelled as a double point source. Of the $3 \mathrm{GHz}$-detections among AzTEC16-30, all the other SMGs except AzTEC21a were found to be unresolved by Miettinen et al. (2015) in the $\sim 1$ 1'. 8 resolution PdBI $1.3 \mathrm{~mm}$ images. A Gaussian fit to AzTEC21a yielded a rather poorly constrained deconvolved FWHM of $\left(2^{\prime \prime} 6 \pm 1^{\prime \prime} .2\right) \times\left(0{ }^{\prime} 3 \pm 00^{\prime} \cdot 5\right)$. For a fair comparison with the present $3 \mathrm{GHz}$ size, we fitted the source using JMFIT, and obtained a $1.3 \mathrm{~mm}$ FWHM size of $2^{\prime \prime} .79_{-0.71}^{+0.66} \times 00^{\prime} 60_{-0.60}^{+0.59}\left(\mathrm{PA}=47^{\circ} .8_{-15.3}^{+11.0}\right)$, which is comparable to the aforementioned value, and the major axis is about $2.1_{-0.9}^{+1.7}$ times larger than that at $3 \mathrm{GHz}\left(1^{\prime \prime} .34_{-0.44}^{+0.40}\right)$. AzTEC21a is potentially a blend of smaller (sub)mm-emitting sources (Miettinen et al. 2015), hence appears more extended at $1.3 \mathrm{~mm}$ than its radio size. We note that the PdBI $1.3 \mathrm{~mm}$ emission of AzTEC27 could not be well modelled by a single Gaussian source model; the major axis FWHM was determined to be $\theta_{\text {maj }}=3$ ". 6 (Miettinen et al. 2015). Similarly 
Table 5. Rest-frame FIR/submm and UV/optical sizes of our $3 \mathrm{GHz}$ detected SMGs.

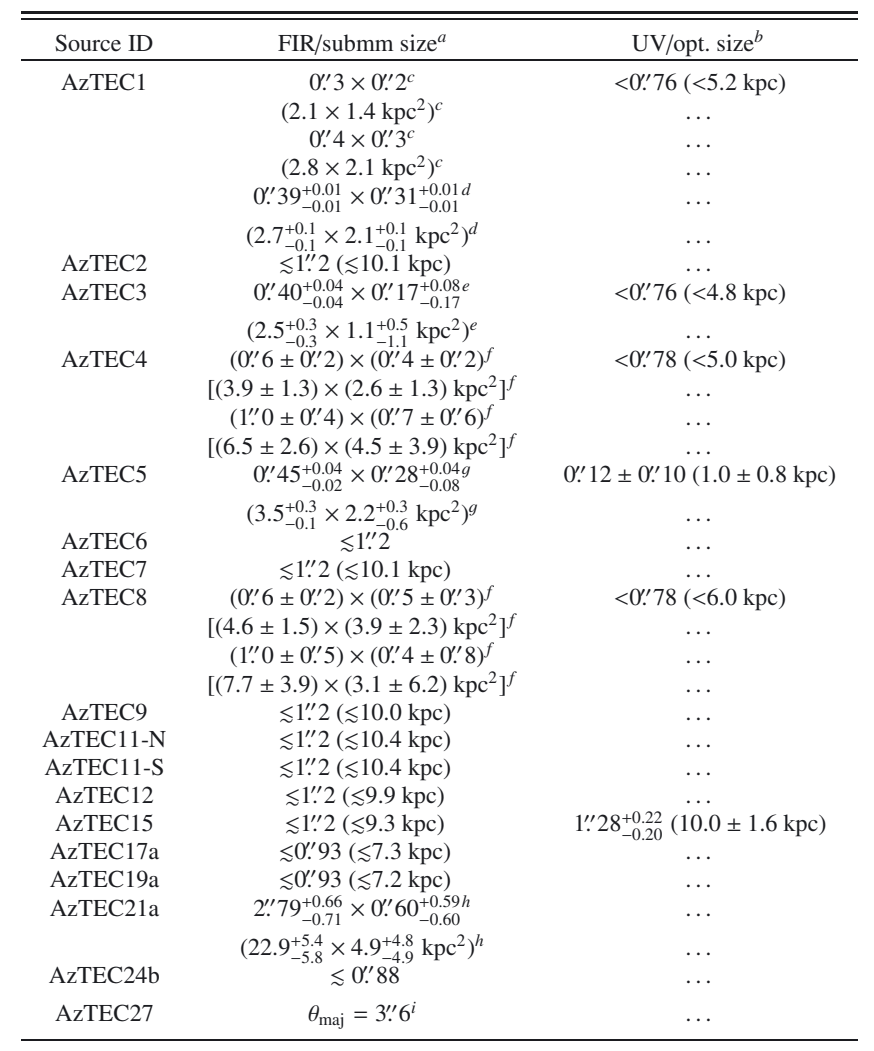

Notes. Besides the angular sizes, the physical sizes are given in parentheses when a spectroscopic or photometric redshift is available. ${ }^{(a)}$ The upper FWHM size limits for AzTEC2-15 at observed-frame $890 \mu \mathrm{m}$ are from Younger et al. (2007, 2009), while those for AzTEC17a, 19a, and 24b refer to observed-frame $1.3 \mathrm{~mm}$ (Miettinen et al. 2015) and represent half of the beam major axis FWHM. ${ }^{(b)}$ The diameter at restframe UV/optical derived from the effective radii from Toft et al. (2014; see text for details). ${ }^{(c)}$ The FWHM size derived from SMA $890 \mu$ m data by Younger et al. (2008) when modelling the source as a Gaussian (upper value) or elliptical disk (lower value). ${ }^{(d)}$ The FWHM size measured from the ALMA $870 \mu \mathrm{m}$ image (Miettinen et al., in prep.) using JMFIT. (e) A deconvolved FWHM size derived through ALMA 1 mm observations by Riechers et al. (2014). ${ }^{(f)}$ The FWHM size derived from SMA $870 \mu \mathrm{m}$ data by Younger et al. (2010) when modelling the source as a Gaussian (upper value) or elliptical disk (lower value). ${ }^{(g)}$ The FWHM size measured from the ALMA $994 \mu$ m image (Miettinen et al., in prep.) using JMFIT. ${ }^{(h)}$ The observed-frame $1.3 \mathrm{~mm}$ FWHM size of AzTEC21a derived here using JMFIT. ${ }^{(i)}$ The major axis FWHM at $1.3 \mathrm{~mm}$ from Miettinen et al. (2015).

to AzTEC21a, AzTEC27 could be a blend of more compact sources. Higher-resolution (sub)mm imaging is required to examine the possible multiplicity of AzTEC21a and 27. The constraints on the (sub)mm FWHM sizes of our SMGs are listed in Table 5 and plotted against their $3 \mathrm{GHz}$ major axis FWHM sizes in Fig. 5.

In addition to AzTEC1, 3, 4, 5, and 8, Toft et al. (2014) also derived the rest-frame UV/optical sizes for AzTEC10 and AzTEC15 (see their Table 1). The measurements were based on UltraVISTA observations. The diameters were found to be $1.4 \pm 0.2 \mathrm{kpc}\left(0{ }^{\prime} 18_{-0.04}^{+0.02}\right)$ for AzTEC10, and $10.0 \pm 1.6 \mathrm{kpc}$ $\left(1^{\prime \prime} .28_{-0.20}^{+0.22}\right)$ for AzTEC15. The $3 \mathrm{GHz}$ major axis FWHM of AzTEC15 $\left(\theta_{\text {maj }}=1^{\prime \prime} .21_{-0.32}^{+0.29}\right)$ is in good agreement with its UV/optical extent, while AzTEC10 was not detected at $3 \mathrm{GHz}$.

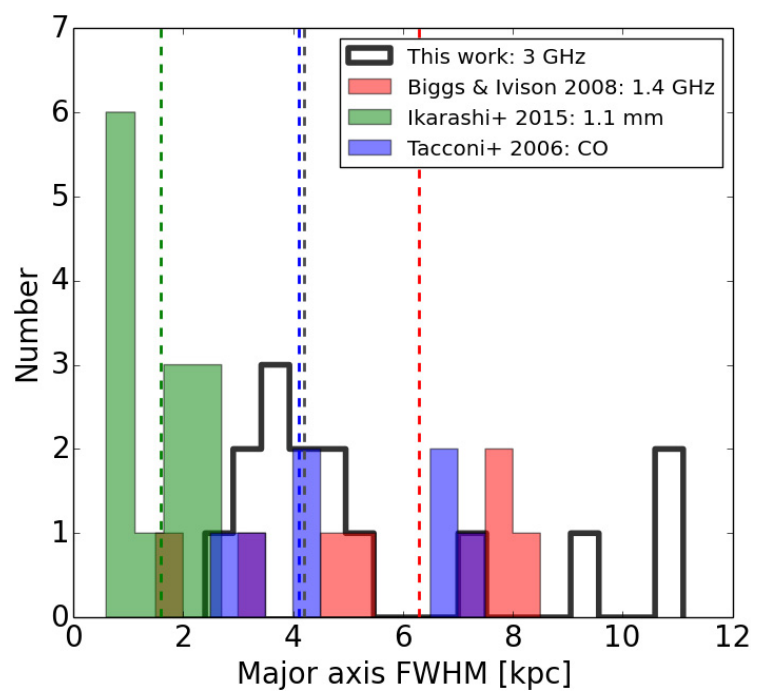

Fig. 6. Distribution of the major axis FWHM sizes of our COSMOS SMGs as seen at $v_{\mathrm{obs}}=3 \mathrm{GHz}$ is shown by an open histogram. For comparison, the following SMG major axis size distributions are also shown: 1.4 GHz sizes from Biggs \& Ivison (2008), $1.1 \mathrm{~mm}$ sizes from Ikarashi et al. (2015), and the extent of CO molecular gas in the SMGs studied by Tacconi et al. (2006). The upper size limits were placed in the bins corresponding to those values. The vertical dashed lines show the corresponding median major axis sizes (4.2 kpc for AzTEC1-21a, $6.3 \mathrm{kpc}$ for the Biggs \& Ivison (2008) SMGs, $1.6 \mathrm{kpc}$ for the Ikarashi et al. (2015) SMGs, and $4.1 \mathrm{kpc}$ for the CO sizes from Tacconi et al. (2006); survival analysis was used to take the upper size limits into account when calculating the median sizes). See text for details.

We note that heavy obscuration by dust can lead to an apparent compact size at the rest-frame UV/optical wavelengths. However, one would expect the central galactic regions to be more extincted compared to the outer portions, which could affect the surface brightness profile in such a way that the measured size (e.g. the half-light radius) is larger than in the case of no differential dust extinction. However, if the extincted outer parts of a galaxy fall below the detection limit, the effect might go in the opposite direction.

\subsection{Comparison to SMG sizes from the literature}

In this subsection, we discuss the SMG sizes derived in previous surveys at different wavelengths. The measured sizes discussed below are derived using the following four observational probes: i) radio continuum emission at centimetre wavelengths; ii) (sub)mm continuum emission (typically corresponding to rest-frame FIR); iii) molecular spectral line emission arising from rotational transitions of $\mathrm{CO}$; and iv) rest-frame optical emission tracing the spatial extent of the stellar content. A selection of size distributions derived from the reported data in the studies discussed below is shown in Fig. 6 alongside with our $3 \mathrm{GHz}$ size distribution.

\subsubsection{Radio sizes}

A previous work of immediate interest for comparison with our results is the MERLIN/VLA $1.4 \mathrm{GHz}$ survey $(1 \sigma=$ $6 \mu \mathrm{Jy}$ beam $^{-1}$; $\sim 0.52 \times 0$. '48 resolution) by Biggs \& Ivison (2008) of the Lockman Hole SMGs (spanning a redshift range of $z_{\text {spec }}=1.147-2.689$ ). The median deconvolved FWHM size we derived from their data (their Table 3 of AIPS/JMFIT-derived 
sizes) is $\left(0{ }^{\prime} 61 \pm 0 .{ }^{\prime} 10\right) \times\left(0.31 \pm 0{ }^{\prime} 08\right)^{5}$. This is comparable to our median size of $\left(0{ }^{\prime} 54 \pm 00^{\prime} 11\right) \times\left(0{ }^{\prime} 35 \pm 0{ }^{\prime}(04)\right.$ derived from 2.1 times higher frequency observations. The median linear size from Biggs \& Ivison (2008), $(6.3 \pm 0.9) \times(3.3 \pm 0.7) \mathrm{kpc}^{2}$ (scaled to the Planck 2015 cosmology), is $1.5 \pm 0.4$ times larger in the major axis than our value of $(4.2 \pm 0.9) \times(2.3 \pm 0.4) \mathrm{kpc}^{2}$. If we consider only those SMGs in our sample that lie in the redshift range studied by Biggs \& Ivison (2008), i.e. AzTEC7, 11$\mathrm{N}, 11-\mathrm{S}$, and 12, the discrepancy becomes more significant: the median linear major axis of these four sources is $3.5 \pm 0.5 \mathrm{kpc}$, i.e. $1.8 \pm 0.4$ times smaller than that from Biggs \& Ivison (2008). However, given the relatively small number of sources in these two (sub)samples $\left(z_{\text {spec }}\right.$ values are available for eight SMGs in the Biggs \& Ivison 2008 sample), the latter comparison might be susceptible to small number statistics.

To see how the millimetre flux densities of the Biggs \& Ivison (2008) SMGs compare to those of our SMGs, we compiled their Bolocam 1.1 mm (Laurent et al. 2005), MAMBO $1.2 \mathrm{~mm}$ (Ivison et al. 2005), and SCUBA $850 \mu \mathrm{m}$ (Ivison et al. 2007) flux densities and converted them to $1.1 \mathrm{~mm}$ flux densities assuming that $\beta=1.5$ when needed. The resulting flux density range is $S_{1.1 \mathrm{~mm}}=1.4_{-0.7}^{+0.6}-6.0_{-1.4}^{+1.4} \mathrm{mJy}$, which includes somewhat fainter sources than ours with the JCMT/AzTEC $1.1 \mathrm{~mm}$ flux densities of $S_{1.1 \mathrm{~mm}}=3.3_{-1.6}^{+1.4}-9.3_{-1.3}^{+1.3} \mathrm{mJy}$ (Scott et al. 2008). However, the two $1.1 \mathrm{~mm}$ flux density ranges are comparable within the uncertainties, and hence the radio size comparison is reasonable. Moreover, we found no correlation between the Biggs \& Ivison (2008) SMGs' radio sizes and their millimetre flux densities (cf. our Fig. 3, bottom panel). We note that Chapman et al. (2004), who studied 12 Hubble Deep Field SMGs $(z=1.01-2.91)$ using the MERLIN/VLA $1.4 \mathrm{GHz}$ observations $(0 . ' 2-0 . ' 3$ resolution), found that in most cases $(67 \pm 24 \%)$ the radio emission is resolved on angular scales of $\sim 1^{\prime \prime}(\sim 8.5 \mathrm{kpc}$ at their median redshift of $z=2.2$ ). The median diameter (measured above $3 \sigma$ emission) was reported to be $0 .{ }^{\prime} 83 \pm 0 . ' 14$ $\left(7.0_{-1.4}^{+1.2} \mathrm{kpc}\right)$, which is larger than our median $3 \mathrm{GHz}$ major axis FWHM by a factor of $1.5 \pm 0.4$, although it was not specified whether the median diameter refers to the deconvolved FWHM as determined in the present paper (we note that according to Biggs \& Ivison 2008, the size reported by Chapman et al. 2004 is the largest extent within the $3 \sigma$ contour, hence not directly comparable with our FWHM sizes). The authors concluded that their SMGs are extended starbursts and therefore different from local ULIRGs with sub-kpc nuclear starburst regions (e.g. Condon et al. 1991). Biggs et al. (2010) used $18 \mathrm{~cm}$ Very Long Baseline Interferometry (VLBI) radio observations at a very high angular resolution of about 30 mas to examine the sizes of a sample of six compact SMGs drawn from the Biggs \& Ivison (2008) sample. Only two of these six SMGs $(33 \pm 6 \%)$ were found to host an ultra-compact AGN radio core, and the authors concluded that the radio emission from their SMGs is mostly arising from star formation rather than from an AGN activity.

\subsection{2. (Sub)mm sizes}

Ikarashi et al. (2015) recently derived a size distribution for a sample of 13 high-redshift $\left(z_{\text {phot }} \sim 3-6\right)$ SMGs through $1.1 \mathrm{~mm}$ ALMA observations at $\sim 0.2$ resolution. Their SMGs were originally discovered in the ASTE/AzTEC $1.1 \mathrm{~mm}$ observations of the Subaru/XMM-Newton Deep Field (S. Ikarashi et al., in prep.),

\footnotetext{
The median sizes from other works that we report in this section were derived (when relevant) using a survival analysis as described in Sect. 4.1.
}

and the reported ALMA $1.1 \mathrm{~mm}$ flux densities lie in the range of $S_{1.1 \mathrm{~mm}}=(1.23 \pm 0.07)-(3.45 \pm 0.10) \mathrm{mJy}$. Compared to the JCMT/AzTEC $1.1 \mathrm{~mm}$ flux densities of our SMGs, namely $S_{1.1 \mathrm{~mm}}=3.3_{-1.6}^{+1.4}-9.3_{-1.3}^{+1.3} \mathrm{mJy}$ (Scott et al. 2008), the Ikarashi et al. (2015) SMGs are fainter, which hampers the direct comparison of these two SMG samples. Ikarashi et al. (2015) measured the sizes using the $u v$ visibility data directly, and assumed symmetric Gaussian profiles. From the values given in their Table 1 we derived a median FWHM of $0 .{ }^{\prime} 22 \pm 0 . ' 04$. The linear radii reported by the authors are very compact, $\sim 0.3-1.3 \mathrm{kpc}$ (median $\sim 0.8 \mathrm{kpc}$ ), which translate to diameters of $\sim 0.6-2.6 \mathrm{kpc}$ (median $\sim 1.6 \mathrm{kpc}$ ). These sizes suggest that the high-redshift $(z \gtrsim 3)$ SMGs are associated with a compact starburst region (as seen at $\lambda_{\text {rest }}<160-289 \mu \mathrm{m}$ ), and Ikarashi et al. (2015) concluded that the median SFR surface density of their SMGs, $\sim 10^{2} M_{\odot} \mathrm{yr}^{-1} \mathrm{kpc}^{-2}$, is comparable to that of local merger-driven (U)LIRGs and higher than those of low- and high- $z$ (extended) disk galaxies.

Simpson et al. (2015a) carried out a high-resolution $(0.35 \times$ $0.25) 870 \mu \mathrm{m}$ ALMA survey of a sample of 30 of the brightest $850 \mu \mathrm{m}$-selected SMGs from the SCUBA-2 Cosmology Legacy Survey of the UKIDSS Ultra Deep Survey (UDS) field. Their target SMGs have $850 \mu \mathrm{m}$ flux densities of $S_{850 \mu \mathrm{m}}=8-16 \mathrm{mJy}$ $\left(>4 \sigma ; S_{1.1 \mathrm{~mm}}=3.2-6.5 \mathrm{mJy}\right.$ if $\left.\beta=1.5\right)$, and hence are mostly comparable to our flux-limited sample (only three of our SMGs lie above this flux density range). For a subsample of 23 SMGs (detected at $S / N_{870 \mu \mathrm{m}}>10$ ), they derived a median size of $0.30 \pm 0 . ' 04(2.4 \pm 0.2 \mathrm{kpc})$ for the major axis FWHM through Gaussian fits in the $u v$ plane $^{6}$. The authors pointed out that Gaussian fits in the image plane yielded sizes consistent with those derived in the $u v$ plane, the median ratio between the two being $F W H M(u v) / F W H M$ (image) $=0.9 \pm 0.2$. Given the photo- $z$ values of the Simpson et al. (2015a) SMGs, the derived median size refers to that at $\lambda_{\text {rest }} \sim 250 \mu \mathrm{m}$. The median angular (linear) size at a comparable rest-frame wavelength from Ikarashi et al. (2015) is still $\sim 36 \%(\sim 50 \%)$ smaller than in the Simpson et al. (2015a) survey. On the other hand, our median $3 \mathrm{GHz}$ angular (linear) major axis FWHM is $1.8 \pm 0.4(1.8 \pm 0.5)$ times larger than the median observed-frame $870 \mu \mathrm{m}$ size from Simpson et al. (2015a). Similarly, Simpson et al. (2015a) concluded that their rest-frame FIR sizes are considerably smaller ( $\sim 2$ times on average) than the $1.4 \mathrm{GHz}$ radio-continuum sizes from Biggs \& Ivison (2008).

\subsubsection{Size of the $\mathrm{CO}$ emission}

In Fig. 6, we also show the sizes of SMGs as derived through high-resolution $(\sim 0.6) \mathrm{CO}$ spectral line $(J=3-2$ and 7-6) observations with the PdBI by Tacconi et al. (2006). We derived a median CO-emitting FWHM size of $\left(0{ }^{\prime} 40 \pm 0 . ' 12\right) \times$ $\left(0{ }^{\prime} 40 \pm 0 . ' 10\right)$ or $(4.1 \pm 1.0) \times(3.3 \pm 0.9) \mathrm{kpc}^{2}$ for the Tacconi et al. (2006) SMGs that lie at $z_{\text {spec }}=2.202-2.509$ and have a reported circular/elliptical Gaussian-fit ( $u v$ plane) FWHM size in their Table 1. We note that the target SMGs of Tacconi et al. (2006) have SCUBA $850 \mu \mathrm{m}$ flux densities of $S_{850 \mu \mathrm{m}}=8.2-10.7 \mathrm{mJy}$, which correspond to $1.1 \mathrm{~mm}$ flux densities of $S_{1.1 \mathrm{~mm}} \sim 3.3-4.3 \mathrm{mJy}$ (assuming $\beta=1.5$ ). Hence, in terms of $S_{1.1 \mathrm{~mm}}$, those SMGs are comparable to

6 Simpson et al. (2015a) do not tabulate the individual source sizes, and hence we cannot plot the corresponding $\lambda_{\mathrm{obs}}=870 \mu \mathrm{m}$ size distribution in our Fig. 6. We note that Simpson et al. (2015b) list the angular FWHM sizes of these ALMA SMGs in their Table 1, but the source redshifts are not tabulated by Simpson et al. (2015a,b). 
the faintest sources in our sample (AzTEC21-30) where we have only three $3 \mathrm{GHz}$ detections (AzTEC21a, 24b, and 27). Moreover, three of the Tacconi et al. (2006) SMGs appear to be hosting an AGN (SMM J044307+0210, J123549+6215, and J123707+6214). Nevertheless, our median $3 \mathrm{GHz}$ major axis FWHM appears to be comparable to the median CO-emission major axis FWHM from Tacconi et al. (2006): the ratio between the two in angular and linear units is $1.4 \pm 0.5$ and $1.0 \pm 0.3$, respectively. The mid- to high- $J \mathrm{CO}$ lines observed by Tacconi et al. (2006) are more sensitive to denser and warmer molecular gas than lower excitation $\left(J_{\text {up }} \leq 2\right)$ lines, and therefore the total molecular extent is expected to be larger. Indeed, one of the Tacconi et al. (2006) SMGs (J123707+6214) was observed in $\mathrm{CO}(J=1-0)$ with the VLA by Riechers et al. (2011), and it was found to be more spatially extended than that seen in $\mathrm{CO}(3-2)$ emission. Engel et al. (2010; Table 1 therein) provided a compilation of different $\mathrm{CO}$ rotational transition observations towards SMGs, and reported linear HWHM sizes for the SMGs as derived using circular Gaussian fits in the $u v$ plane (with two exceptions where the quoted size corresponds to the half-light radius). Their target SMGs are characterised by $850 \mu \mathrm{m}$ flux densities of $S_{850 \mu \mathrm{m}} \geq 5 \mathrm{mJy}\left(S_{1.1 \mathrm{~mm}} \geq 2 \mathrm{mJy}\right.$ if $\left.\beta=1.5\right)$, and this threshold is exceeded by all our SMGs in the JCMT/AzTEC $1.1 \mathrm{~mm}$ survey (Scott et al. 2008). From their data we derived a median HWHM value of $1.85 \pm 0.39 \mathrm{kpc}$, which corresponds to a FWHM of $3.70 \pm 0.78 \mathrm{kpc}$, consistent with the aforementioned size we calculated for the Tacconi et al. (2006) SMGs, and hence comparable to our median radio emission size (the median major axis FWHM being $4.2 \pm 0.9 \mathrm{kpc}$ ).

\subsubsection{The spatial extent of the stellar emission}

Chen et al. (2015) studied the rest-frame optical sizes of SMGs. Based on the Hubble/WFC3 observations of a sample of 48 ALMA-detected $z=1-3$ SMGs in the Extended Chandra Deep Field South (ALESS SMGs), the authors measured a median effective radius (half-light radius along the semimajor axis within which half of the total flux is emitted) of $4.4_{-0.5}^{+1.11} \mathrm{kpc}$ through fitting a Sérsic profile to the $H_{160}$-band $\left(\lambda_{\text {pivot }}=1536.9 \mathrm{~nm}\right)$ surface brightness of each SMG. Simpson et al. (2015a) compared their FIR sizes to the optical sizes from Chen et al. (2015), and found a large difference of about a factor of four between the two (the optical emission being more extended). Interestingly, the median radius at the rest-frame UV/optical for the AzTEC SMGs from Toft et al. (2014) is only $0.7 \mathrm{kpc}$ (derived using survival analysis; see our Table 5 for the diameters), but we note that most of these sources are very highredshift SMGs (such as AzTEC1 and AzTEC3), which makes their size determination more difficult, and, as mentioned in Sect. 5.1, the measured sizes are probably subject to strong dust extinction.

\section{Discussion}

\subsection{The spatial extent of SMGs as seen in the radio, dust, gas, and stellar emission}

The radio continuum emission, thermal dust emission, and molecular spectral line emission can all be linked to the stellar evolution process in a galaxy. Star formation takes place in molecular clouds where the gas and dust are well mixed. The molecular gas content is best traced by the rotational line emission of CO. However, different transitions (arising from different
$J$ levels) have different excitation characteristics, hence are probing regions of differing physical and chemical properties: the high-excitation line emission is arising from denser and warmer phase, while low-excitation lines (especially the $J=1-0$ transition) are probing colder, more spatially extended gas reservoirs (e.g. Ivison et al. 2011; Riechers et al. 2011). Dust grains absorb the UV/optical photons emitted by the young, newly formed stellar population, and then re-emit the absorbed energy in the FIR. When the high-mass stars undergo SN explosions, the associated blast waves and remnant shocks give rise to synchrotron radio emission produced by relativistic CRs. This connection is believed to lead to the tight FIR-radio correlation (see Sect. 1 and references therein). On the basis of this connection, one would also expect the FIR- and radio-emission size scales to be similar. The galactic-scale outflows driven by the starburst phenomenon (SNe, stellar winds, and radiation pressure) are not expected to overcome the gravitational potential of the galaxy, hence not dispersing the ISM out of the galaxy (this requires a stronger feedback from the AGN; e.g. Tacconi et al. 2006). To summarise, the radio continuum, rest-frame FIR, and mid- to high- $J$ CO transitions are all expected to trace regions of active star formation, and hence the corresponding spatial extents of their emission are expected to be comparable to each other. However, as the size comparison in the previous subsection shows, this does not seem to be the case for SMGs.

As discussed in Sect. 5.2, we have found that the $3 \mathrm{GHz}$ radio-continuum sizes are comparable to the $\mathrm{CO}$-emission sizes from Tacconi et al. (2006) and Engel et al. (2010), but more extended than the FIR emission seen in other studies, most notably when compared to those from Ikarashi et al. (2015). A possible scenario is that SMGs have a two-component ISM: a spatially extended gas component, which is traced by low- to mid- $J$ CO line emission and radio continuum emission, and a more compact starburst region giving rise to the higher- $J$ CO line emission. In the former component, a low dust temperature would lead to a low dust luminosity, while the latter one - having an elevated dust temperature - could dominate the luminosityweighted dust continuum size measurements (cf. Riechers et al. 2011).

Simpson et al. (2015a) suggested that the larger radiocontinuum size compared to that at rest-frame FIR is the result of CR diffusion in the galactic magnetic field (e.g. Murphy et al. 2008). To quantify this, they convolved their median $870 \mu \mathrm{m}$ size with an exponential kernel and a scale length of 1-2 kpc on the basis of the diffusion length of CR electrons in local starforming galaxies (which is an order of magnitude longer than the mean free path of dust-heating UV photons; Bicay \& Helou 1990; Murphy et al. 2006, 2008). The convolved size (FWHM) of 3.8-5.2 kpc they derived is in better agreement with the median major axis FWHM of $6.3 \pm 0.9 \mathrm{kpc}$ from Biggs \& Ivison (2008; see our Sect. 5.2). However, as pointed out by Simpson et al. (2015a), the diffusion scale length of CRs in SMGs might be shorter than the aforementioned value because of the higher SFR surface density in SMGs (Murphy et al. 2008; see our Appendix E).

The rest-frame FIR sizes of AzTEC1 and AzTEC3 (see Sect. 5.1) suggest that they are comparable to their $3 \mathrm{GHz}$ radio sizes within the uncertainties, but higher-resolution (sub)mm continuum imaging of all our SMGs is required to better constrain their FIR emission sizes, and to examine whether they represent the population of very compact SMGs, similarly to those from Ikarashi et al. (2015). However, even if the radio size is more extended than the FIR emission, the short cooling time of CR electrons in starburst galaxies $\left(\sim 10^{4}-10^{5} \mathrm{yr}\right)$ suggests that 
their diffusion through the ISM to spatial scales larger than FIR emission is infeasible (see Appendix E for the calculation; the diffusion length ranges from only a few tens of pc to $\sim 10^{2} \mathrm{pc}$ ). Hence, the CR diffusion scenario proposed by Simpson et al. (2015a) seems unlikely, and in Sect. 6.2 we will discuss a possible alternative explanation for a more extended radio emission in SMGs.

A further puzzle is the fact that the rest-frame FIR sizes of SMGs appear smaller than the CO-emitting size given the FIR$\mathrm{CO}$ correlation found for different types of galaxies at both lowand high-z, including SMGs (see e.g. Carilli \& Walter 2013, for a review; Fig. 7 therein). The large difference found between the rest-frame FIR and optical sizes of SMGs (about a factor of four; see our Sect. 5.2.4) led Simpson et al. (2015a) to conclude that the spatial extent of ongoing star formation is more compact than the spatial distribution of pre-existing stellar population, and that their SMGs might be undergoing a period of bulge growth. As pointed out by Chen et al. (2015), if the highredshift $(z \gtrsim 3)$ SMGs are progenitors of $z \sim 2$ compact, quiescent galaxies (cQGs; see Toft et al. 2014), the high- $z$ SMGs have to go through a major transformation to decrease the spatial extent of the stellar component (and to increase the Sérsic index) before being quenched.

\subsection{Merger-induced extended synchrotron emission}

In the scenario where the FIR size of a galaxy is smaller than its radio-emitting region, and where $\mathrm{CR}$ electron diffusion - due to rapid radiative cooling $\left(\sim 10^{4}-10^{5} \mathrm{yr}\right)-$ is unlikely to be the reason for a more extended radio size (which is potentially the case here; see Appendix E), an alternative interpretation is required. One possibility is that if SMGs are driven by mergers (e.g. Tacconi et al. 2008; Engel et al. 2010), the interacting progenitor disk galaxies can perturb each others magnetic fields by pulling them out to larger spatial scales (see Murphy 2013). Hence, a significant amount of non-star formation related radio emission can arise from the merger system. Murphy (2013) concluded that this taffy-like merger scenario could explain the low FIR/radio ratios and steep high-frequency radio spectra of local compact starbursts and those seen in some high- $z$ SMGs. In this scenario, mergers are expected to be associated with stretched magnetic field structures between the colliding galaxies, giving rise to synchrotron bridges between them and/or tidal tails (Condon et al. 1993). The synchrotron-emitting relativistic electrons in such bridges might have their origin in merger-induced shock acceleration, rather than having travelled there from the progenitor galaxies due to the rapid cooling time (Lisenfeld \& Völk 2010; Murphy 2013; see also Donevski \& Prodanovic 2015).

The $3 \mathrm{GHz}$ sources investigated here are fairly centrally concentrated and no evidence of interaction-induced bridges/tails is seen except towards AzTEC1, 2, and AzTEC11. There is a $\sim 2.6 \sigma 3 \mathrm{GHz}$ feature lying 1". 5 to the NE of AzTEC1, and the $3 \mathrm{GHz}$ major axis FWHM of AzTEC1 $\left(0{ }^{\prime} 67_{-0.20}^{+0.17}\right)$ is larger than the sample median major axis FWHM $\left(0{ }^{\prime} 54^{-0} 0{ }^{\prime} \cdot 11\right)$. AzTEC2 exhibits an additional $3 \mathrm{GHz}$ source to the $\mathrm{SW}$, which might be an indication of a merging pair (or a radio jet). The additional source has a major axis FWHM of $0.2_{-0.44}^{+0.31}$, which is also larger than the median $\theta_{\text {maj }}$ of $0{ }^{\prime} 54 \pm 0 . ' 11$. The two $3 \mathrm{GHz}$ components seen towards AzTEC11 share a common $3 \sigma 3 \mathrm{GHz}$ envelope, but AzTEC11-N and 11-S both have a $3 \mathrm{GHz}$ major axis FWHM size smaller than the median value $\left(0 .^{\prime} 40_{-0.08}^{+0.07}\right.$ and $\left.0 .^{\prime} 48_{-0.05}^{+0.04}\right)$. The $1.4 \mathrm{GHz}$ morphologies of the Biggs \& Ivison (2008; their Fig. 3) SMGs are generally more elongated and clumpy than our

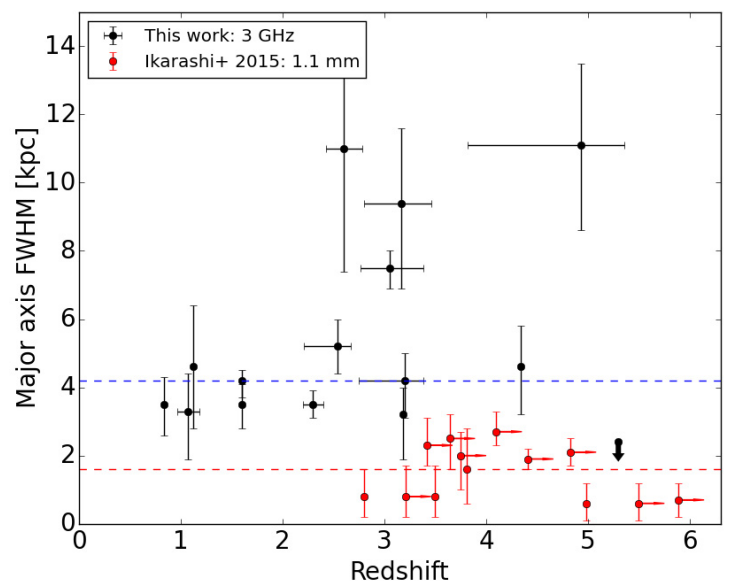

Fig. 7. Linear major axis FWHM sizes [kpc] of our AzTEC SMGs at $3 \mathrm{GHz}$ plotted as a function of redshift. The upper size limit of AzTEC3 is indicated by a downward pointing arrow. Also shown (red points) are the $1.1 \mathrm{~mm}$ sizes from Ikarashi et al. (2015). The horizontal blue and red dashed lines show the corresponding median major axis FWHM values of $4.2 \mathrm{kpc}$ and $1.6 \mathrm{kpc}$, respectively.

sources, which could suggest a higher merger fraction among their SMGs, and hence somewhat more extended radio emission sizes (see Sect. 5.2.1). However, a fair fraction of our target SMGs ( $\sim 36 \%$ of the total sample) show clumpy/disturbed morphologies or evidence of close companions at different wavelengths (Younger et al. 2007, 2009; Toft et al. 2014; Miettinen et al. 2015), which could be manifestations of galaxy mergers.

To conclude, there could be a possible connection between merger-driven SMGs and their larger radio-emitting size as compared to FIR emission, as would be expected if the above described merger scenario is true. However, the spatial distribution of molecular gas, as traced by mid- to high- $J$ lines, appears to be comparable to the $v_{\mathrm{obs}}=3 \mathrm{GHz}$ radio emission size. As described in Sect. 6.1, this is to be expected if the observed radio size of a galaxy is a direct tracer of its spatial extent of star formation. This would not be consistent with the scenario where the CRs emit synchrotron radiation as a result of processes not related to star formation, such as the aforementioned merger scenario. However, these comparisons between $\mathrm{CO}$ and radio median sizes are, unfortunately, based on measurements obtained from different samples and the result can be affected by subtle selection effects. For example, the $1.4 \mathrm{GHz}$ radio sizes from Biggs \& Ivison (2008) are instead larger than the CO sizes from Tacconi et al. (2006; see our Fig. 6), which is qualitatively consistent with the scenario of merger-induced synchrotron emission. To quantitatively compare the spatial extents of radio emission and molecular gas component, high-resolution radio and $\mathrm{CO}$ imaging of the same sample of SMGs is required.

\subsection{Size evolution as a function of redshift and the effect of galaxy environment}

In Fig. 7, we show our deconvolved linear major axis FWHM sizes as a function of redshift. No statistically significant correlation can be seen between these two quantities, which is consistent with that found by Simpson et al. (2015a) and Chen et al. (2015) at shorter wavelengths. We note, however that, with the exception of AzTEC3 (see below), there is a hint of larger radio sizes at $z \sim 2.5-5$ compared to our lower redshift SMGs: the $z \sim 2.5-5$ SMGs tend to lie above the median size of our sample (4.2 kpc, blue dashed line). Also plotted in Fig. 7 are 
the $1.1 \mathrm{~mm}$ FWHM sizes from Ikarashi et al. (2015). These authors discussed that the compact sizes of their high-redshift $\left(z_{\text {phot }} \sim 3-6\right)$ SMGs support the scenario where they represent the precursors of cQGs seen at $z \sim 2$, which, in turn, are believed to evolve into the massive ellipticals seen in the presentday $(z=0)$ universe (Toft et al. 2014). We note that among the Ikarashi et al. (2015) SMG sample, the $\lambda_{\mathrm{obs}}=1.1 \mathrm{~mm}$ sizes are larger at $z \sim 3.5-5$ than those outside that redshift range, although it should be noted that most of their SMGs in this $z$ range have only lower $z$ limits available. As mentioned above, there is some resemblance in our data, i.e. the radio sizes appear larger at a comparable redshift range of $z \sim 2.5-5$.

Ikarashi et al. (2015) discussed that if both the radio and FIR continuum are tracers of star-forming regions, then the $z \gtrsim 3$ SMGs are more compact than the lower-redshift SMGs typically observed in radio continuum emission (e.g. Biggs \& Ivison 2008). As shown in Fig. 7, our present VLA $3 \mathrm{GHz}$ data do not suggest such a trend, and, as mentioned earlier, there is actually a hint of larger radio sizes at $z \sim 2.5-5$ compared to lower redshifts. However, the highest-redshift SMG in our sample, AzTEC3 at $z \simeq 5.3$, shows the most compact size among our sources, consistent with the rest-frame FIR sizes from Ikarashi et al. (2015). We note that Capak et al. (2011) found that AzTEC3 belongs to a spectroscopically confirmed protocluster containing eight galaxies within a $1 \operatorname{arcmin}^{2}$ area, and therefore the environment might also play a role in the galaxy size evolution (see also Smolčić et al. 2015b). However, it is currently unclear whether the environmental effects in a galaxy overdensity will lead to a more compact or more extended radioemitting size than field galaxies. On one side, a protocluster environment is expected to show an elevated merger rate (e.g. Hine et al. 2015), and, as discussed above, mergers are expected to pull the galactic magnetic fields to larger spatial scales, and hence lead to a more extended radio synchrotron emission. On the other side, the ram and/or thermal pressures of the intracluster medium could compress the ISM of the galaxy, increase the magnetic field strength, and hence cause an excess in radio emission (consistent with a low IR-radio $q$ parameter of $\lesssim 2$ for AzTEC3; Miettinen et al., in prep.). The aforementioned pressure forces can drive shock waves into the ISM, and hence accelerate the CR particles (Murphy 2009). Consequently, the cooling time and diffusion length-scale of CR electrons can decrease (see Appendix E), resulting in a compact radio-emitting area. More detailed environmental analysis of SMGs is needed to understand this further.

\section{Summary and conclusions}

We have used radio-continuum observations taken by the VLACOSMOS $3 \mathrm{GHz}$ Large Project to study the radio sizes of a sample of SMGs originally detected with the AzTEC bolometer array at $1.1 \mathrm{~mm}$, and all followed up with (sub)mm interferometric observations. Our main results are summarised as follows:

1. Of the total sample of $39 \mathrm{SMGs}, 3 \mathrm{GHz}$ emission was detected towards 18 or $\sim 46 \pm 11 \%$ of them $(S / N=4.2-37.4)$. Four sources (AzTEC2, 5, 8, and 11) show two separate $3 \mathrm{GHz}$ sources.

2. The median angular radio-emitting size (FWHM) we derived is $\left(0 \prime^{\prime} 54 \pm 0 \prime^{\prime} 11\right) \times\left(0 \prime^{\prime} 35 \pm 00^{\prime} 04\right)$. In linear units, derived for the SMGs with known redshift, we obtained a median size of $(4.2 \pm 0.9) \times(2.3 \pm 0.4) \mathrm{kpc}^{2}$. The low brightness temperature values of $T_{\mathrm{B}}=1.4 \pm 0.7 \mathrm{~K}$ to $75.9 \pm 16.6 \mathrm{~K}$ are consistent with the radio emission being powered by star formation, rather than by an AGN.

3. We found no obvious correlations between the FWHM radio-size and radio or submm flux density or redshift, which is consistent with previous studies at other wavelengths.

4. We found that our derived $3 \mathrm{GHz}$ sizes are comparable to $1.4 \mathrm{GHz}$ and $\mathrm{CO}$-emission sizes of SMGs reported in literature, yet they are $\sim 1.7-2.8$ times larger than the median rest-frame FIR sizes based on high-resolution ALMA observations, and reported in literature (see Sect. 5.2 for details).

5. If both the radio and FIR continuum are tracing the same regions of star formation in a galaxy as expected from the FIRradio correlation, then the differing spatial scales of these emissions is puzzling. A possible explanation is that SMGs have a two-component ISM: i) an extended gas component with a low dust temperature, which gives rise to the lowto mid- $J$ CO line and radio continuum emissions; and ii) a warmer, compact starburst region giving rise to the high$J$ rotational line emission of $\mathrm{CO}$, which could dominate the dust continuum size measurements. The more extended radio-emitting size with respect to the compact FIR-emitting size was suggested to be the result of cosmic-ray diffusion by Simpson et al. (2015a). However, we have shown here that the short electron cooling times of $\sim 10^{4}-10^{5} \mathrm{yr}$ in dense starburst galaxies do not allow the electrons to spread away from their sites of origin to the required spatial scales. Hence, it seems more probable that the observed synchrotron emission partly originates in regions around the active starburst region, possibly from extended magnetic fields driven by the galaxy merging process.

Acknowledgements. We thank the referee for constructive comments that helped to improve this paper. This research was funded by the European Union's Seventh Framework programme under grant agreement 337595 (ERC Starting Grant, “CoSMass"). M.A. acknowledges partial support from FONDECYT through grant 1140099. A.K. acknowledges support by the Collaborative Research Council 956, sub-project A1, funded by the Deutsche Forschungsgemeinschaft (DFG). This paper makes use of the following ALMA data: ADS/JAO.ALMA\#2012.1.00978.S and ADS/JAO.ALMA\#2013.1.00118.S. ALMA is a partnership of ESO (representing its member states), NSF (USA) and NINS (Japan), together with NRC (Canada), NSC and ASIAA (Taiwan), and KASI (Republic of Korea), in cooperation with the Republic of Chile. The Joint ALMA Observatory is operated by ESO, AUI/NRAO and NAOJ. This research has made use of NASA's Astrophysics Data System, and the NASA/IPAC Infrared Science Archive, which is operated by the JPL, California Institute of Technology, under contract with the NASA. We greatfully acknowledge the contributions of the entire COSMOS collaboration consisting of more than 100 scientists. More information on the COSMOS survey is available at http://www. astro. caltech. edu/ cosmos

\section{References}

Aretxaga, I., Wilson, G. W., Aguilar, E., et al. 2011, MNRAS, 415, 3831 Barger, A. J., Cowie, L. L., Sanders, D. B., et al. 1998, Nature, 394, 248 Beck, R., \& Golla, G. 1988, A\&A, 191, L9

Bell, E. F. 2003, ApJ, 586, 794

Bicay, M. D., \& Helou, G. 1990, ApJ, 362, 59

Biggs, A. D., \& Ivison, R. J. 2008, MNRAS, 385, 893

Biggs, A. D., Younger, J. D., \& Ivison, R. J. 2010, MNRAS, 408, 342

Blain, A. W., Smail, I., Ivison, R. J., et al. 2002, Phys. Rep., 369, 111

Bondi, M., Ciliegi, P., Schinnerer, E., et al. 2008, ApJ, 681, 1129

Bourke, S., Mooley, K., \& Hallinan, G. 2014, Astronomical Data Analysis Software and Systems XXIII, 485, 367

Capak, P. L., Riechers, D., Scoville, N. Z., et al. 2011, Nature, 470, 233

Carilli, C. L., \& Walter, F. 2013, ARA\&A, 51, 105

Carilli, C. L., \& Yun, M. S. 1999, ApJ, 513, L13

Carilli, C. L., \& Yun, M. S. 2000, ApJ, 530, 618

Casey, C. M., Narayanan, D., \& Cooray, A. 2014, Phys. Rep., 541, 45

Chapman, S. C., Smail, I., Windhorst, R., et al. 2004, ApJ, 611, 732 
Chen, C.-C., Smail, I., Swinbank, A. M., et al. 2015, ApJ, 799, 194

Condon, J. J. 1992, ARA\&A, 30, 575

Condon, J. J., Huang, Z.-P., Yin, Q. F., \& Thuan, T. X. 1991, ApJ, 378, 65

Condon, J. J., Helou, G., Sanders, D. B., \& Soifer, B. T. 1993, AJ, 105, 1730

Crutcher, R. M. 1999, ApJ, 520, 706

da Cunha, E., Walter, F., Smail, I., et al. 2015, ApJ, 806, 110

Donevski, D., \& Prodanović, T. 2015, MNRAS, 453, 638

Downes, A. J. B., Peacock, J. A., Savage, A., \& Carrie, D. R. 1986, MNRAS, 218, 31

Dumas, G., Schinnerer, E., Tabatabaei, F. S., et al. 2011, AJ, 141, 41

Engel, H., Tacconi, L. J., Davies, R. I., et al. 2010, ApJ, 724, 233

Fu, H., Cooray, A., Feruglio, C., et al. 2013, Nature, 498, 338

González, J. E., Lacey, C. G., Baugh, C. M., \& Frenk, C. S. 2011, MNRAS, 413, 749

Hainline, L. J., Blain, A. W., Smail, I., et al. 2009, ApJ, 699, 1610

Helou, G., Soifer, B. T., \& Rowan-Robinson, M. 1985, ApJ, 298, L7

Helsel, D. R. 2005, Nondetects And Data Analysis: Statistics for Censored Environmental Data (New York: John Wiley and Sons)

Hine, N. K., Geach, J. E., Alexander, D. M., et al. 2015, MNRAS, submitted [arXiv: 1506.05115 ]

Ho, L. C., \& Ulvestad, J. S. 2001, ApJS, 133, 77

Hughes, D. H., Serjeant, S., Dunlop, J., et al. 1998, Nature, 394, 241

Ikarashi, S., Ivison, R. J., Caputi, K. I., et al. 2015, ApJ, 810, 133

Ivison, R. J., Smail, I., Dunlop, J. S., et al. 2005, MNRAS, 364, 1025

Ivison, R. J., Greve, T. R., Dunlop, J. S., et al. 2007, MNRAS, 380, 199

Ivison, R. J., Papadopoulos, P. P., Smail, I., et al. 2011, MNRAS, 412, 1913

Lacki, B. C., \& Beck, R. 2013, MNRAS, 430, 3171

Lacki, B. C., \& Thompson, T. A. 2010, ApJ, 717, 196

Laurent, G. T., Aguirre, J. E., Glenn, J., et al. 2005, ApJ, 623, 742

Lisenfeld, U., \& Völk, H. J. 2010, A\&A, 524, A27

Lisenfeld, U., Völk, H. J., \& Xu, C. 1996, A\&A, 314, 745

Magnelli, B., Lutz, D., Santini, P., et al. 2012, A\&A, 539, A155

McMullin, J. P., Waters, B., Schiebel, D., et al. 2007, Astronomical Data Analysis Software and Systems XVI, 376, 127

Miettinen, O., Smolčić, V., Novak, M., et al. 2015, A\&A, 577, A29

Morić, I., Smolčić, V., Kimball, A., et al. 2010, ApJ, 724, 779

Mundell, C. G., Wilson, A. S., Ulvestad, J. S., \& Roy, A. L. 2000, ApJ, 529, 816

Murphy, E. J. 2009, ApJ, 706, 482

Murphy, E. J. 2013, ApJ, 777, 58

Murphy, E. J., Helou, G., Braun, R., et al. 2006, ApJ, 651, L111

Murphy, E. J., Helou, G., Kenney, J. D. P., et al. 2008, ApJ, 678, 828

Murphy, E. J., Kenney, J. D. P., Helou, G., et al. 2009, ApJ, 694, 1435
Murphy, E. J., Porter, T. A., Moskalenko, I. V., et al. 2012a, ApJ, 750, 126 Murphy, E. J., Bremseth, J., Mason, B. S., et al. 2012b, ApJ, 761, 97 Murphy, E. J., Stierwalt, S., Armus, L., et al. 2013, ApJ, 768, 2

Novak, M., Smolčić, V., Civano, F., et al. 2015, MNRAS, 447, 1282

Planck Collaboration XIII. 2015, A\&A, submitted [arXiv: 1502 . 01589]

Rau, U., \& Cornwell, T. J. 2011, A\&A, 532, A71

Riechers, D. A., Capak, P. L., Carilli, C. L., et al. 2010, ApJ, 720, L131

Riechers, D. A., Carilli, L. C., Walter, F., et al. 2011, ApJ, 733, LL11

Riechers, D. A., Carilli, C. L., Capak, P. L., et al. 2014, ApJ, 796, 84

Roseboom, I. G., Bunker, A., Sumiyoshi, M., et al. 2012, MNRAS, 426, 1782

Salvato, M., Ilbert, O., Hasinger, G., et al. 2011, ApJ, 742, 61

Sargent, M. T., Schinnerer, E., Murphy, E., et al. 2010, ApJS, 186, 341

Schinnerer, E., Smolčić, V., Carilli, C. L., et al. 2007, ApJS, 172, 46

Schinnerer, E., Sargent, M. T., Bondi, M., et al. 2010, ApJS, 188, 384

Scott, D., \& Tout, C. A. 1989, MNRAS, 241, 109

Scott, K. S., Austermann, J. E., Perera, T. A., et al. 2008, MNRAS, 385, 2225

Scoville, N., Aussel, H., Brusa, M., et al. 2007, ApJS, 172, 1

Simpson, J. M., Swinbank, A. M., Smail, I., et al. 2014, ApJ, 788, 125

Simpson, J. M., Smail, I., Swinbank, A. M., et al. 2015a, ApJ, 799, 81

Simpson, J. M., Smail, I., Swinbank, A. M., et al. 2015b, ApJ, 807, 128

Smail, I., Ivison, R. J., \& Blain, A. W. 1997, ApJ, 490, L5

Smolčić, V., Aravena, M., Navarrete, F., et al. 2012, A\&A, 548, A4

Smolčić, V., Karim. A., Miettinen, O., et al. 2015a, A\&A, 576, A127

Smolčić, V., Miettinen, O., Tomičić, N. et al. 2015b, A\&A, submitted

Swinbank, A. M., Chapman, S. C., Smail, I., et al. 2006, MNRAS, 371, 465

Swinbank, A. M., Simpson, J. M., Smail, I., et al. 2014, MNRAS, 438, 1267

Tabatabaei, F. S., Beck, R., Krause, M., et al. 2007, A\&A, 466, 509

Tacconi, L. J., Neri, R., Chapman, S. C., et al. 2006, ApJ, 640, 228

Tacconi, L. J., Genzel, R., Smail, I., et al. 2008, ApJ, 680, 246

Toft, S., Smolčić, V., Magnelli, B., et al. 2014, ApJ, 782, 68

Urquhart, J. S., Hoare, M. G., Purcell, C. R., et al. 2009, A\&A, 501, 539

Völk, H. J., Aharonian, F. A., \& Breitschwerdt, D. 1996, Space Sci. Rev., 75, 279

Xu, C., Klein, U., Meinert, D., Wielebinski, R., \& Haynes, R. F. 1992, A\&A 257,47

Younger, J. D., Fazio, G. G., Huang, J.-S., et al. 2007, ApJ, 671, 1531

Younger, J. D., Fazio, G. G., Wilner, D. J., et al. 2008, ApJ, 688, 59

Younger, J. D., Fazio, G. G., Huang, J.-S., et al. 2009, ApJ, 704, 803

Younger, J. D., Fazio, G. G., Ashby, M. L. N., et al. 2010, MNRAS, 407, 1268

Yun, M. S., Reddy, N. A., \& Condon, J. J. 2001, ApJ, 554, 803

Yun, M. S., Aretxaga, I., Gurwell, M. A., et al. 2015, MNRAS, 454, 3485 


\section{Appendix A: Notes on peculiar $3 \mathrm{GHz}$ sources}

AzTEC2, 5, and 8 each show, in addition to a $3 \mathrm{GHz}$ source coinciding with the submm peak, an additional source lying at 1.'51 SW, 6.'56 NE, and 2.'62 NE from the SMA peak, respectively. The additional $3 \mathrm{GHz}$ feature towards AzTEC2 could, in principle, represent a radio-emitting lobe of a jet interacting with the surrounding medium or a merger component (projected separation is 12.7 proper kpc at the redshift of AzTEC2). We note that the $1.3 \mathrm{~mm}$ emission detected towards AzTEC2 with ALMA also shows an additional weak $(\sim 2.9 \sigma)$ feature at 2 '. 14 to the SW of AzTEC2 (Cycle 2 ALMA project 2013.1.00118.S; M. Aravena et al., in prep.), but its peak position lies 0.'60 away to the SW of the $3 \mathrm{GHz}$ feature; this offset is within the large statistical positional uncertainty of $\sim 1^{\prime \prime}$ of the weak $1.3 \mathrm{~mm}$ feature $\left(\Delta \theta_{\text {stat }} \propto(S / N)^{-1}\right)$.

The additional radio source towards AzTEC5 was already seen at $1.4 \mathrm{GHz}$ (see Table 4), and it is also visible in the Spitzer/IRAC and MIPS images (Younger et al. 2007, Fig. 1 therein). This source can be associated with the Herscheldetected emission-line galaxy $150.08336+02.53619$, for which a spectroscopic redshift of $z_{\text {spec }}(\mathrm{H} \alpha)=1.42$ was reported by Roseboom et al. (2012). Also, a 5.4 $\sigma$ detection with ALMA at $994 \mu \mathrm{m}$ is obtained towards this source (Cycle 1 ALMA project 2012.1.00978.S; PI: A. Karim; O. Miettinen et al., in prep.), while the ALMA $1.3 \mathrm{~mm}$ detection is of $\sim 3 \sigma$ significance (M. Aravena et al., in prep.). We note that the most up-todate COSMOS spec- $z$ catalogue gives a lower redshift value of $z_{\text {spec }}=0.9044$, which is based on observations with the Inamori Magellan Areal Camera and Spectrograph (IMACS; Salvato et al., in prep.); however, the quality flag is 1 , i.e. this $z_{\text {spec }}$ is considered insecure. Hence, the $3 \mathrm{GHz}$ source NE of AzTEC5 is probably a lower-redshift galaxy (AzTEC5 itself has $z_{\text {phot }}=$ $3.05_{-0.28}^{+0.33}$; see Table 1). Similarly, the two radio sources towards AzTEC 8 were already seen at $1.4 \mathrm{GHz}$ (Younger et al. 2009, Fig. 1 therein; see our Table 4): the $1.4 \mathrm{GHz}$ source to the NE of the SMA-detected SMG was called AzTEC8.E by Younger et al. (2009), and the Spitzer $24 \mu \mathrm{m}$ emission towards AzTEC8 is coincident with AzTEC8.E. This source can be associated with the Chandra/X-ray -detected galaxy CXOC J095959.5+023441 whose photo- $z$ is $z_{\text {phot }}=2.420 \pm 0.060$ (Salvato et al. 2011), hence it is probably at a lower redshift than the SMG on its western side $\left(z_{\text {spec }}=3.179\right)$. This source is also detected with the VLBA at $1.4 \mathrm{GHz}$ at milliarcsec resolution $\left(S_{1.4 \mathrm{GHz}}=83.8 \mu \mathrm{Jy}\right)$, showing the presence of a radio-emitting AGN (Herrera Ruiz et al., in prep.; Herrera Ruiz, priv. comm.).

Towards AzTEC11, we have detected a double $3 \mathrm{GHz}$ source, projectively separated by 1 '. 5 , and where the southern component is coincident with AzTEC11-N ${ }^{7}$. The northern $3 \mathrm{GHz}$ source is nearly equidistant from AzTEC11-N (1".38 separation) and AzTEC11-S (1".40 separation). Younger et al. (2009) reported that the calibrated visibility data of AzTEC11 show significant structure and are best modelled with a double point source (their Table 2). The complex structure of the visibility data probably makes the derived source positions to be rather uncertain. However, a positional uncertainty of only 0.2 in both right ascension and declination for the $890 \mu \mathrm{m}$ peak of AzTEC11-N and 11-S was reported by Younger et al. (2009; Table 1 therein). The authors also recognised an elongated $1.4 \mathrm{GHz}$ source towards AzTEC11, where the emission morphology resembles that seen at $890 \mu \mathrm{m}$. Their two-component

\footnotetext{
7 We note that the southern SMA source towards AzTEC11 was accidentally called AzTEC11.N by Younger et al. (2009), while the northern component was called AzTEC11.S (see Table 1 in Younger et al. 2009).
}

Gaussian fit yielded comparable $1.4 \mathrm{GHz}$ flux densities for the two sources (see our Table 4). The two $3 \mathrm{GHz}$ sources seen towards AzTEC11 share a common radio envelope (at the $3 \sigma$ level), and are probably in the process of merging ${ }^{8}$. The ALMA $1.3 \mathrm{~mm}$ observations at $1 . .36 \times 0.78$ resolution towards AzTEC11 revealed two SMGs separated by 1". 45 in projection (M. Aravena et al., in prep.), and the northern one is well coincident with our northern $3 \mathrm{GHz}$ source (only 0.' 11 offset).

Towards AzTEC15 the projected separation between the $890 \mu \mathrm{m}$ and $3 \mathrm{GHz}$ positions is fairly large, i.e. $1^{\prime \prime}$. 19 . The $890 \mu \mathrm{m}$ detection of AzTEC15 by Younger et al. (2009) was only of moderate significance $(4.4 \sigma)$, and no $1.4 \mathrm{GHz}$ counterpart was detected, but it was found to be associated with Spitzer IR emission. The $890 \mu \mathrm{m}$ position uncertainty reported by Younger et al. (2009) is $0 ! 3$ in right ascension and 0.2 in declination. The 1 ". $36 \times 0$.'78 resolution ALMA $1.3 \mathrm{~mm}$ observations towards AzTEC15 (M. Aravena et al., in prep.), however, show a perfect positional coincidence ( 0. '03 offset) with our $3 \mathrm{GHz}$ source of $5.4 \sigma$ significance, and hence it is physically related to AzTEC15.

The $3 \mathrm{GHz}$ source near AzTEC21a (1". $15 \mathrm{NE}$ of the PdBI position) is our weakest source with a $\mathrm{S} / \mathrm{N}$ of 4.2. The reliability of this $3 \mathrm{GHz}$ source candidate is supported by the fact that it is also seen at $1.4 \mathrm{GHz}$, although the $1.4 \mathrm{GHz}$ source is also weak (peak surface brightness of $63 \mu \mathrm{Jy}$ beam $^{-1}$ or $\sim 3.9 \sigma$; see Miettinen et al. 2015, Fig. A.1 therein). Hence, we include the $3 \mathrm{GHz}$ source near AzTEC21a in our radio size analysis.

The $3 \mathrm{GHz}$ source $(12.6 \sigma)$ detected 2.'67 SW in projection from AzTEC24b is also detected at $1.4 \mathrm{GHz}$ (Table 4), and the $1.4 \mathrm{GHz}$ source was associated with the ASTE/AzTEC $1.1 \mathrm{~mm}$ SMG AzTEC/C48 by Aretxaga et al. (2011), a source also detected with Herschel (see Miettinen et al. 2015). There is also a $1.3 \mathrm{~mm}$-detected ALMA source lying 2".63 SW from our PdBI detection and 0.'26 SE from the $3 \mathrm{GHz}$ source in question (Aravena et al., in prep.). The ALMA detection in particular shows that the $3 \mathrm{GHz}$ source is an SMG despite the relatively large separation from our PdBI source. We note that there is a $4.3 \sigma 3 \mathrm{GHz}$ source lying 0.'47 $\mathrm{N}$ of AzTEC27. Miettinen et al. (2015) reported the presence of weak $1.4 \mathrm{GHz}$ emission (peak intensity of $32.1 \mu \mathrm{Jy}$ beam $^{-1}$ or $\sim 2.5 \sigma$ ) towards this position, but the nature of this radio emission remains unclear (i.e. noise feature or associated with the SMG). The weak $3 \mathrm{GHz}$ source associated with AzTEC27 is included in the present radio size analysis. The additional $3 \mathrm{GHz}$ radio sources not analysed further in the present study are described in Appendix B, and those not detected at $3 \mathrm{GHz}$ are discussed in Appendix C.

\section{Appendix B: Notes on additional $3 \mathrm{GHz}$ sources}

As shown in Fig. 2, 5.'70 SW from AzTEC20, 8".43 NW from AzTEC22, and 5'.51 E from AzTEC23, there is a clearly

\footnotetext{
8 The spectroscopic redshift of $z_{\text {spec }}=1.599$ was measured towards a position, which lies 0.' $6 \mathrm{NE}$ of AzTEC11-N's $3 \mathrm{GHz}$ peak position, and 0.9 SW from that of AzTEC11-S (Salvato et al., in prep.). The physical relation of the $3 \mathrm{GHz}$ sources is supported by the very low probability for a chance association. This can be quantified by calculating the Poissonian random probability, $P=1-\mathrm{e}^{-\pi r^{2} N}$, where $r$ is the projected angular distance of the two sources, and $N$ is the surface number density of sources $\left[\mathrm{deg}^{-2}\right]$ that have flux densities greater than or equal to that of the source in question (Downes et al. 1986; Scott \& Tout 1989). Using the VLA-COSMOS 3 GHz Large Project catalogue (Smolčić et al., in prep.), we estimate that the probability of having a AzTEC11-S type $3 \mathrm{GHz}$ source lying 1".5 away from that of AzTEC11$\mathrm{N}$ is only $P \sim 3.4 \times 10^{-4}$. The null hypothesis of chance association is generally rejected if $P<5 \%$.
} 
detected $3 \mathrm{GHz}$ source $(10.3 \sigma, 22.8 \sigma$, and $5.5 \sigma$, respectively). Interestingly, these $3 \mathrm{GHz}$ sources are closer to the original AzTEC $1.1 \mathrm{~mm}$ positions than to the PdBI source candidates (0.75 E, 2'.73 SW, and 4".25 NE from AzTEC20, 22, and 23; see Scott et al. 2008; Miettinen et al. 2015). Morever, the $3 \mathrm{GHz}$ sources detected towards the AzTEC20, 22, and 23 fields have a Herschel $250 \mu \mathrm{m}$ detection lying at 2.'45 SW, 1.'48 SE, and 7'.78 NW, respectively (as based on the cross-correlation with the COSMOS SPIRE $250 \mu \mathrm{m}$ Photometry Catalogue). These radio sources are not analysed further in the present study.

\section{Appendix C: Notes on the 3 GHz non-detections' appearances at other wavelengths}

The following 21 SMGs $(54 \pm 12 \%$ of the whole sample) were not detected at 3 GHz: AzTEC10, 13, 14-E, 14-W, 16, 17b, 18, 19b, 20, 21b, 21c, 22, 23, 24a, 24c, 26a, 26b, 28, 29a, 29b, and 30.

AzTEC10, 13, 14-E, and 14-W were detected at $890 \mu \mathrm{m}$ with a $\mathrm{S} / \mathrm{N}$ of $5.3,4.6,5.0$, and 3.9, respectively, by Younger et al. (2009). Only the most significant of these moderate SMA $890 \mu$ m detections, namely AzTEC10, was found to exhibit Spitzer IR emission, while none of them was detected at optical wavelengths or at $1.4 \mathrm{GHz}$ (Younger et al. 2009). Hence, their non-detection at $3 \mathrm{GHz}$ is not surprising.

As discussed by Miettinen et al. (2015; Appendix C therein), the PdBI $1.3 \mathrm{~mm}$ SMG candidates AzTEC16, 17b, 20, 21c, $22,24 \mathrm{a}, 24 \mathrm{c}, 26 \mathrm{~b}, 29 \mathrm{a}$, and 30 have no multiwavelength counterparts and some of them could be spurious. The $1.3 \mathrm{~mm}$ $\mathrm{S} / \mathrm{N}$ of these sources were found to be in the range of 4.5-6. Moreover, AzTEC29b, although a $7.3 \sigma$ detection, was found to lie at edge of the $1.3 \mathrm{~mm}$ map. On the other hand, AzTEC27 and AzTEC28 $\left(S / N_{1.3 \mathrm{~mm}}=6\right.$ and 5.5, respectively) were among the best PdBI detections by Miettinen et al. (2015), but neither of them were found to have multiwavelength counterparts; only a trace of $1.4 \mathrm{GHz}$ emission $(2.5 \sigma)$ was seen towards AzTEC27 (Appendix A). AzTEC18, 19b, 21b, 21c, 23, and 26a were detected with $S / N_{1.3 \mathrm{~mm}}=4.2-9.7$, and only AzTEC21c was found to not have multiwavelength counterparts. However, none of these SMGs was detected at $1.4 \mathrm{GHz}$. Given the aforementioned properties, it comes as no surprise that among AzTEC16-30 there are so many $3 \mathrm{GHz}$ non-detections ( 17 of 22 , i.e. $77 \pm 19 \%$ ).

\section{Appendix D: Testing the reliability of the size measurements}

To test the reliability of our FWHM size measurements, we simulated sources using the CASA (release 4.3.1) Toolkit. We first generated mock galaxies with a Gaussian flux distribution, intrinsic FWHM size fixed to $0.74 \times 0$ '. 45 (i.e. the average deconvolved FWHM derived for our SMGs where both the major and minor axes could be determined or constrained), PA ranging from $0^{\circ}$ to $135^{\circ}$ in steps of $45^{\circ}$, and flux densities corresponding to $\mathrm{S} / \mathrm{N}$ in the range of 4 to 38 in steps of $S / N=2$, which cover the observed range of $\mathrm{S} / \mathrm{N}$ of our $\mathrm{SMGs}(S / N=4.2-37.4)$. The simulated image was convolved to the resolution of 0.75 to match the resolution of our real data. To obtain a realistic background noise level, the simulated galaxies were added to a $3 \mathrm{GHz}$ map of $1.5 \times 1.5$ in size, and which was cropped from a sourcefree region of COSMOS $\left(1 \sigma=2.3 \mu \mathrm{Jy}_{\text {beam }}{ }^{-1}\right)$. The resulting image is shown in the top panel in Fig. D.1. The deconvolved FWHM sizes of the simulated sources were then determined using the AIPS task JMFIT as described in Sect. 4.1.
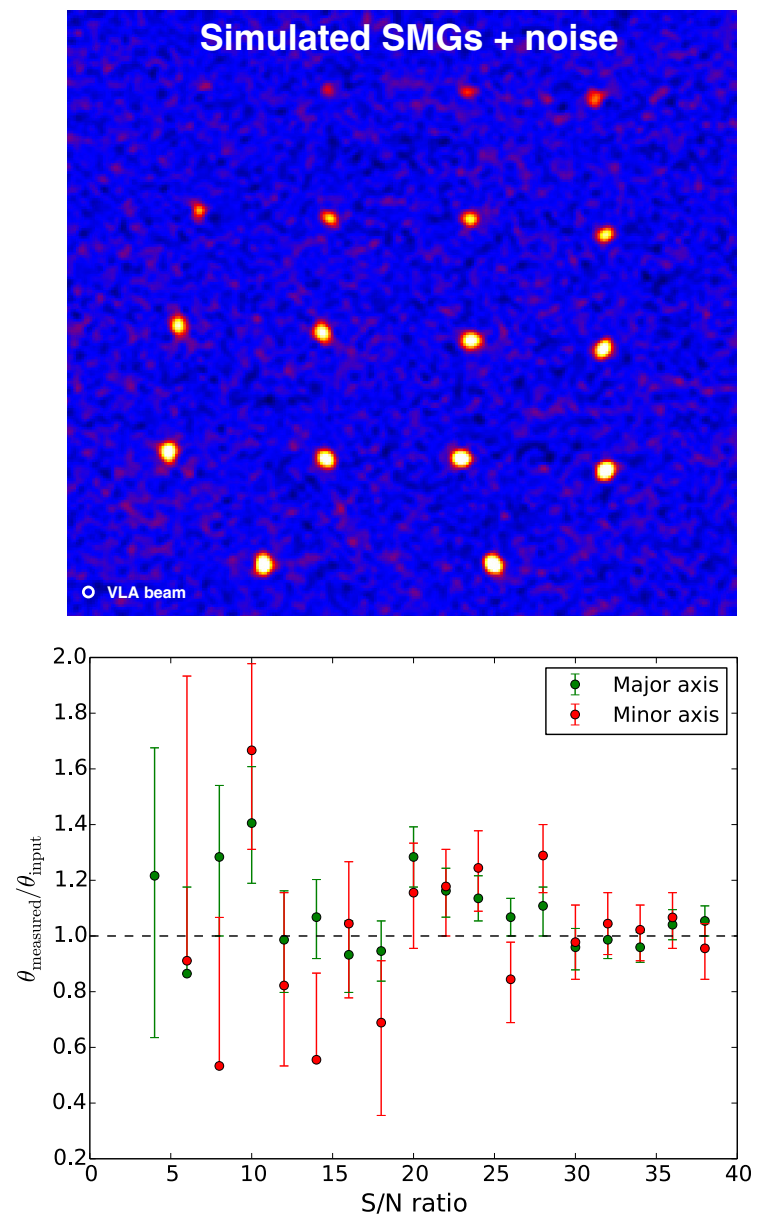

Fig. D.1. Top: simulated SMGs added to a noise map to simulate our real $3 \mathrm{GHz}$ VLA data. The $\mathrm{S} / \mathrm{N}$ of the sources increases from left to right, top to bottom $(S / N=4-38)$, and in each row the PA ranges from $0^{\circ}$ to $135^{\circ}$ in steps of $45^{\circ}$ (being $0^{\circ}$ and $45^{\circ}$ for the two bottommost

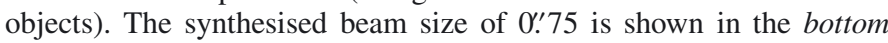
left corner. Bottom: ratio of the measured to the simulated input source size (deconvolved FWHM) as a function of the $\mathrm{S} / \mathrm{N}$. The green points show the major axes ratio, while the red points show that between the minor axes. The minor axis FWHM for the faintest source $(S / N=4)$ could not be determined by the AIPS task JMFIT. The vertical error bars were propagated from the size errors determined by JMFIT. A onesided error bar is shown for those cases where the minimum size could not be determined by JMFIT. The horizontal dashed line shows the line of equality between the sizes.

The bottom panel in Fig. D.1 shows the ratio of the measured size to the input size as a function of the $\mathrm{S} / \mathrm{N}$. The data points are shown separately for the major and minor axes. As expected, the size measurement is generally more accurate for more significant detections, but within the size uncertainties determined by JMFIT the measured deconvolved sizes are in good agreement with the real intrinsic sizes (see the dashed line in Fig. D.1 indicating the one-to-one correspondence). Because most of our detections are of high significance (median $S / N=12.6$ ), these simulations suggest that our size measurements are reliable.

\section{Appendix E: Calculation of the cosmic-ray electron cooling times}

To quantitatively examine the possibility that the observed radiocontinuum sizes of SMGs could be the result of CR diffusion, 
we first calculate the maximum lifetime of the electrons considering the radiative energy losses due to synchrotron emission, inverse Compton (IC) scattering, bremsstrahlung, and ionisation processes. In what follows, we calculate the corresponding cooling times using the formulas from Murphy (2009; Sect. 2 therein) to which we refer the reader for a more detailed description.

The redshifts of our $3 \mathrm{GHz}$ detected SMGs range from $z_{\text {spec }}=0.834$ for AzTEC17a to $z_{\text {spec }}=5.298$ for AzTEC3. If we assume that the critical frequency at which the electron emits most of its energy, $v_{\text {crit }}$, is equal to $v_{\text {rest }}=v_{\text {obs }}(1+z)$, its value is in the range of $5.5 \mathrm{GHz}$ to $18.9 \mathrm{GHz}$. If the electrons are spiralling in a magnetic field whose strength is $B \sim 100 \mu \mathrm{G}$ (a starbursttype $B$-field; e.g. Lacki \& Beck 2013), the relationship $v_{\text {crit }}=$ $1.3 \times 10^{-2}(B / \mu \mathrm{G})(E / \mathrm{GeV})^{2}$ yields $\mathrm{CR}$ electron energies of $E \simeq 2-3.8 \mathrm{GeV}$. For this case, the synchrotron cooling timescale for CR electrons is $\tau_{\text {syn }} \sim 1.4 \times 10^{9}\left(v_{\text {crit }} / \mathrm{GHz}\right)^{-1 / 2}(B / \mu \mathrm{G})^{-3 / 2} \sim$ $3.2-6 \times 10^{5} \mathrm{yr}$.

The IC cooling timescale is given by $\tau_{\text {IC }} \sim 5.7 \times$ $10^{7}\left(v_{\text {crit }} / \mathrm{GHz}\right)^{-1 / 2}(B / \mu \mathrm{G})^{1 / 2}\left(U_{\text {rad }} / 10^{-12} \mathrm{erg} \mathrm{cm}^{-3}\right)^{-1} \mathrm{yr}$, where $U_{\text {rad }}$ is the radiation field energy density of the galaxy. To estimate the value of $U_{\text {rad }}$, we adopt a total infrared $(8-1000 \mu \mathrm{m})$ luminosity range of $L_{\mathrm{IR}} \sim 10^{12}-10^{13} L_{\odot}$ appropriate for SMGs (e.g. Magnelli et al. 2012; Swinbank et al. 2014; da Cunha et al. 2015; see Miettinen et al., in prep., for the present SMG sample), and as the characteristic size we use the median $3 \mathrm{GHz}$ major axis FWHM size derived here, i.e. $4.2 \mathrm{kpc}$, which corresponds to a circular area of $13.9 \mathrm{kpc}^{2}$. Using Eq. (4) of Murphy et al. (2012a), these values imply $U_{\text {rad }}$ in the range of $\sim 5 \times 10^{-10}-4.9 \times 10^{-9} \mathrm{erg} \mathrm{cm}^{-3}$; for a smaller IR-emitting area $\left(A_{\mathrm{IR}}\right)$, the value of $U_{\mathrm{rad}} \propto A_{\mathrm{IR}}^{-1}$ would be higher. The values of $v_{\text {crit }}$ and $B$ being as above, we derive $\tau_{\mathrm{IC}} \sim 2.6 \times 10^{4}-4.9 \times 10^{5} \mathrm{yr}$. In the context of IC cooling, it should be noted that our SMG sample contains high-redshift sources, the most extreme case being AzTEC3 at $z_{\mathrm{spec}}=5.298$. At high redshifts, the IC scattering between relativistic electrons and the cosmic microwave background $(\mathrm{CMB})$ - boosting the CMB photon energy - becomes more important compared to the low $z$ universe. The reason for this is that the energy density of the CMB increases steeply with redshift, namely $U_{\mathrm{CMB}} \propto(1+z)^{4}$. For instance, at $z_{\text {spec }}=5.298$, the CMB photon bath has about 140 times higher energy density than that at the lowest-redshift SMG in our sample (AzTEC17a at $\left.z_{\text {spec }}=0.834\right)$. This means that besides the intense IR radiation field in a starburst, IC losses off the CMB photons have the potential to increase the cooling rate of the CR electrons (e.g. Lacki \& Thompson 2010).
The bremsstrahlung lifetime is $\tau_{\text {brem }} \sim 8.6 \times 10^{7}\left(n_{\mathrm{H}} /\right.$ $\left.\mathrm{cm}^{-3}\right)^{-1} \mathrm{yr}$, where $n_{\mathrm{H}}$ is the hydrogen number density of the ISM. Assuming a typical average density range of $n_{\mathrm{H}}=$ $10^{2}-10^{3} \mathrm{~cm}^{-3}$, we obtain $\tau_{\text {brem }} \sim 8.6 \times 10^{4}-8.6 \times 10^{5} \mathrm{yr}^{9}$.

The timescale for ionisation losses can be written as $\tau_{\text {ion }} \sim 3.6 \times 10^{10}\left(v_{\text {crit }} / \mathrm{GHz}\right)^{1 / 2}(B / \mu \mathrm{G})^{-1 / 2}\left(n_{\mathrm{H}} / \mathrm{cm}^{-3}\right)^{-1} \times$ $\left[3 / 2 \times \ln \left(v_{\text {crit }} / B\right)+49\right]^{-1} \mathrm{yr}$, which under our assumptions lies in the range of $\tau_{\text {ion }} \sim 1.9 \times 10^{5}-3.4 \times 10^{6} \mathrm{yr}$.

Finally, due to the combined energy losses from the aforementioned processes, the effective cooling lifetime for CR electrons is given by

$\tau_{\mathrm{cool}}=\frac{1}{\tau_{\mathrm{syn}}^{-1}+\tau_{\mathrm{IC}}^{-1}+\tau_{\mathrm{brem}}^{-1}+\tau_{\mathrm{ion}}^{-1}}$.

The individual timescales calculated above yield $\tau_{\text {cool }} \sim 1.7 \times$ $10^{4}-1.9 \times 10^{5} \mathrm{yr}$. In the case of random-walk diffusion, the electrons' escape scale-length is given by $l_{\mathrm{esc}}=\left(D_{\mathrm{E}} \tau_{\mathrm{esc}}\right)^{1 / 2}$, and when the diffusion coefficient $D_{\mathrm{E}}$ is in the energy-dependent regime $(E \geq 1 \mathrm{GeV})$, the escape length is given by $l_{\mathrm{esc}} \sim$ $7.1 \times 10^{-4}\left(\tau_{\text {esc }} / \mathrm{yr}\right)^{1 / 2}\left(v_{\text {crit }} / \mathrm{GHz}\right)^{1 / 2}(B / \mu \mathrm{G})^{-1 / 2} \mathrm{kpc}$. During the above derived cooling time $\left(\tau_{\text {esc }}=\tau_{\text {cool }}\right)$ the electrons can travel only $l_{\mathrm{esc}} \sim 22-135 \mathrm{pc}$. Hence, we conclude that if the FIR/star-forming sizes of our SMGs are as compact as those from Simpson et al. (2015a; $2.4 \pm 0.2 \mathrm{kpc}$ in median FWHM) and Ikarashi et al. (2015; 1.6 kpc in median FWHM), it seems unlikely that $\mathrm{CR}$ electrons would have had time to propagate from their sites of origin to the large distances where we observe the $3 \mathrm{GHz}$ emission [the median major axis FWHM size being $4.2 \pm 0.9 \mathrm{kpc}]$. In the above analysis we did not add the effect of the IC scattering off the CMB radiation, although at high redshift it can shorten the electron lifetime and diffusion length scale even more. However, apart from AzTEC3, our Fig. 7 does not show evidence of smaller radio sizes at higher redshifts as expected if the electrons have less time to travel away from their site of origin. This is possibly a manifestation of the fact that in starburst galaxies, at whatever redshift they might be, the local stellar radiation field is intense (cf. the above estimate), and hence the CR electrons can suffer from strong IC losses from stellar light besides/instead from the CMB (e.g. Lisenfeld et al. 1996; Lacki \& Thompson 2010). Moreover, we have ignored the fact that if the galaxy is associated with a galactic-scale wind, the CR particles in the wind adiabatically lose momentum and energy on the course of the expansion of the wind (e.g. Völk et al. 1996). The effect of losses due to electron advection would further shorten the diffusion length-scale.

\footnotetext{
9 We note that under the assumption of a magnetic flux freezing, i.e. $B \propto n_{\mathrm{H}}^{1 / 2}$ (e.g. Crutcher 1999), our adopted field strength of $100 \mu \mathrm{G}$ would imply a density of $n_{\mathrm{H}} \simeq 317 \mathrm{~cm}^{-3}$ if $B=10 \mu \mathrm{G}$ at $n_{\mathrm{H}}=1 \mathrm{~cm}^{-3}$ as is typically observed in normal star-forming galaxies (cf. Murphy 2009). Our adopted range of densities brackets this value of $n_{\mathrm{H}}$.
} 